\title{
Article
}

\section{Influence of microvascular sutures on shear strain rate in realistic pulsatile flow}

Wain, Richard, Smith, D J, Hammond, Doug and Whitty, Justin Available at http://clok.uclan.ac.uk/21947/

Wain, Richard ORCID: 0000-0002-8796-0201, Smith, D J, Hammond, Doug and Whitty, Justin ORCID: 0000-0003-1002-5271 (2018) Influence of microvascular sutures on shear strain rate in realistic pulsatile flow. Microvascular Research, 118 . pp. 69-81. ISSN 0026-2862

It is advisable to refer to the publisher's version if you intend to cite from the work. http://dx.doi.org/10.1016/j.mvr.2018.03.001

For more information about UCLan's research in this area go to

http://www.uclan.ac.uk/researchgroups/ and search for <name of research Group>.

For information about Research generally at UCLan please go to http://www.uclan.ac.uk/research/

All outputs in CLoK are protected by Intellectual Property Rights law, including Copyright law. Copyright, IPR and Moral Rights for the works on this site are retained by the individual authors and/or other copyright owners. Terms and conditions for use of this material are defined in the policies page. 


\title{
Influence of microvascular sutures on shear strain rate in realistic pulsatile flow
}

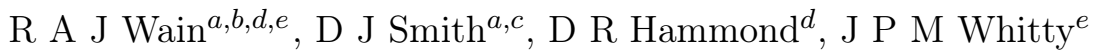 \\ ${ }^{a}$ School of Mathematics, ${ }^{b}$ Institute of Translational Medicine, ${ }^{c}$ Institute for Metabolism and Systems \\ Research, University of Birmingham, B15 2TT, UK. ${ }^{d}$ School of Medicine and Dentistry, \\ ${ }^{e}$ Computational Mechanics Research Group, School of Engineering, University of Central Lancashire, \\ Preston, PR1 2HE, UK
}

\begin{abstract}
Arterial thrombus formation is directly related to the mechanical shear experienced by platelets within flow. High shear strain rates (SSRs) and large shear gradients cause platelet activation, aggregation and production of thrombus. This study, for the first time, investigates the influence of pulsatile flow on local haemodynamics within sutured microarterial anastomoses. We measured physiological arterial waveform velocities experimentally using Doppler ultrasound velocimetry, and a representative example was applied to a realistic sutured microarterial geometry. Computational geometries were created using measurements taken from sutured chicken femoral arteries. Arterial SSRs were predicted using computational fluid dynamics (CFD) software, to indicate the potential for platelet activation, deposition and thrombus formation. Predictions of steady and sinusoidal inputs were compared to analyse whether the addition of physiological pulse characteristics affects local intravascular flow characteristics. Simulations were designed to evaluate flow in pristine and hand-sutured microarterial anastomoses, each with a steady-state and sinusoidal pulse component.

The presence of sutures increased $\mathrm{SSR}_{\max }$ in the anastomotic region by factors of 2.1 and 2.3 in steady-state and pulsatile flows respectively, when compared to a pristine vessel. SSR values seen in these simulations are analogous to the presence of moderate arterial stenosis.

Steady-state simulations, driven by a constant inflow velocity equal to the peak systolic velocity (PSV) of the measured pulsatile flow, underestimated SSRs by $\sim 9 \%$ in pristine, and $\sim 19 \%$ in sutured vessels compared with a realistic pulse. Sinusoidal flows, with equivalent frequency and amplitude to a measured arterial waveform, represent a slight improvement on steady-state simulations, but still SSRs are underestimated by $1-2 \%$. We recommend using a measured arterial waveform, of the form presented here, for simulating pulsatile flows in vessels of this nature.

Under realistic pulsatile flow, shear gradients across microvascular sutures are high, of the order $\sim 7.9 \times 10^{6} \mathrm{~m}^{-1} \mathrm{~s}^{-1}$, which may also be associated with activation of platelets and formation of aggregates.
\end{abstract}

Keywords: Pulsatile; Microvascular; Anastomosis; Sutures; Computational Fluid

Email address: richwain@doctors.org.uk (R A J Wain ${ }^{a, b, d, e}$ ) 
Dynamics (CFD); Shear strain rate.

\section{Corresponding author}

Mr Richard A J Wain, MB ChB MRCS(Eng) MSc(Res) PGCert(Med Ed). Research Fellow, Institute of Translational Medicine and School of Mathematics, University of Birmingham, Edgbaston, B15 2TT, UK.

Email: richwain@doctors.org.uk, Tel: +44 (0)121 414 7374, Fax: +44 (0)121 4143389

\section{Introduction}

Microvascular anastomotic techniques are routine practice in many reconstructive surgical specialties. Common indications include free-tissue transfer for reconstruction following cancer resection or traumatic injury. With state-of-the-art microsurgical equipment and good quality magnification, success rates of free-tissue transfer are often above $95 \%[1,2,3]$. However, often completed in vessels of 1-3 mm diameter using suture material $0.03-0.04 \mathrm{~mm}$ thick, it is a technically challenging procedure with inherent risks and a corresponding failure rate, particularly in non-elective cases $[4,5]$. The majority of failures are attributed to thrombus at the anastomotic site. One factor promoting thrombosis, particularly within arteries, is high intravascular shear strain rate (SSR). Increased SSRs are known to alter the distribution of platelet flow within the blood, cause platelet activation and adherence to vessel walls, and ultimately form thrombus $[6,7]$. SSR is influenced by alterations in blood flow e.g. velocity, and/or changes to the vessel wall surface e.g. due to a pathological stenosis, trauma, or surgery.

In an attempt to combat anastomotic failure secondary to thrombus formation, the Microvascular Anastomotic Coupling (MAC) system (Synovis Micro Companies Alliance, Inc., 439 Industrial Lane, Birmingham, AL 35211, USA) was developed. It has proven to be highly successful, with a complication profile better than hand-sewn venous anastomoses $[8,9]$. It is now widely accepted as routine practice for venous microanastomoses. Coupled microarterial anastomoses however, are not routine in the UK or worldwide, despite some studies demonstrating a degree of success $[10,11]$. The main concerns with arterial coupling are centred around increased wall thickness and reduced pliability when compared to a vein, necessitating a smaller diameter coupler, and introducing technical difficulty in vessel placement. There have also been concerns regarding variable patency with arterial coupling [12].

As venous coupling has become the norm in microsurgical practice, consequently reducing venous thrombosis rates, it could be postulated that a higher proportion of flap failures may be attributed to arterial anastomotic thrombus, although evidence to support this anecdotal experience is not readily available. Nonetheless, hand-sewn anastomoses are standard for arterial repairs, and no particular sutured technique has been shown to be superior, provided the operator adheres to sound microsurgical principles [13].

The relationship between platelet aggregation, hence thrombus formation, and sites of perturbed flow after vascular injury has long been known [14], with intravascular stasis also historically attributed to clot formation [15]. In addition it has been proposed that mechanical shear experienced by platelets within flow causes them to become activated, 
and as such have a propensity to aggregate and form thrombus $[15,16,17,18,19]$. These shear-activated platelets adhere to injured vessel walls and exposed subendothelium in preference to intact, pristine vascular walls $[20,21]$. Also at higher shear rates, red blood cells flow preferentially in the central flow layers, promoting a corresponding relative increase in platelets at the vessel walls, contributing to the rate and extent of arterial thrombus formation [7]. Furthermore, production of the procoagulant fibrin is exaggerated in these high shear environments [22, 23].

Nesbitt et al. [24] suggested that platelets principally use a biomechanical aggregation mechanism at sites of injury, rather than the traditional concept of accumulation of soluble platelet agonists. In their study they propose that the magnitude and spatial distribution of shear micro-gradients directly influences platelet aggregate size [24]. It has been previously demonstrated that sudden high shear promotes membrane tethering between platelets, thus forming aggregates [25]. These platelet tethers then become stronger and more stable when exposed to a subsequent reduction in shear rate, thereby further promoting thrombus growth [24].

Computational Fluid Dynamics (CFD) has previously been used to investigate local blood flow properties, including shear strain rate (SSR), around microvascular anastomoses in an attempt to better understand the biomechanics surrounding different anastomotic techniques [26, 27, 28, 29]. These however, were steady-state continuous flow simulations, thereby neglecting any influence that a pulsatile flow may have on the local haemodynamics. Whilst the use of CFD for simulating vascular flows has increased significantly since initial work in the late 1980s [30, 31, 32, 33], few studies have been carried out investigating microvascular flows, with some notable exceptions [29, 34, 35, 36]. Although well executed, these studies have not examined the influence of clinically representative sutures at the anastomosis, particularly in the context of realistic pulsatile flows. There have however been studies examining both steady [37] and time-dependent $[38,39,40,41]$ blood flows in other vessels throughout the body. Of particular interest was the work of Perdikaris [39] where a range of approaches to simulating cerebral flows was discussed, including the use of dissipative particle dynamics (DPD) to simulate thrombus formation [42]. Whilst DPD is beyond the scope of this study, the technique may prove valuable for future work. This also applies to the more recent study by Tenekecioglu [37], where fluid-structural interactions (FSI) were simulated to investigate the shear stress of coronary arterial endothelium when a bioabsorbable stent was in place. The present study, for the first time, describes the process of applying a measured arterial waveform to a realistic sutured microanastomotic geometry, in an attempt to analyse whether the addition of an authentic pulse affects local intravascular haemodynamics.

\section{Methods}

This study has been designed such that each simulation increases in complexity and is verified prior to embarking on the next stage. Simulations become sequentially more representative of the clinical picture, with specific details of each aspect discussed in the following sections. 


\subsection{Vessel flow rates}

In the same way as for previous work, blood flow velocities were measured using Doppler ultrasonography [27]. A Phillips iU22 ultrasound scanner and corresponding L9-3 probe (Philips Healthcare, 5680 DA Best, The Netherlands) was used to obtain the raw data from preoperative breast reconstruction patients $(n=10)$, with a representative waveform chosen for this study (Figure 1). Calculated means of the vessel diameter and peak systolic velocity (PSV) were used as inlet parameters for the steady-state and idealised sinusoidal simulations. Vessel dimensions and flow rates were provided in isolation for the purposes of this study, with all patient-identifiable information withheld.

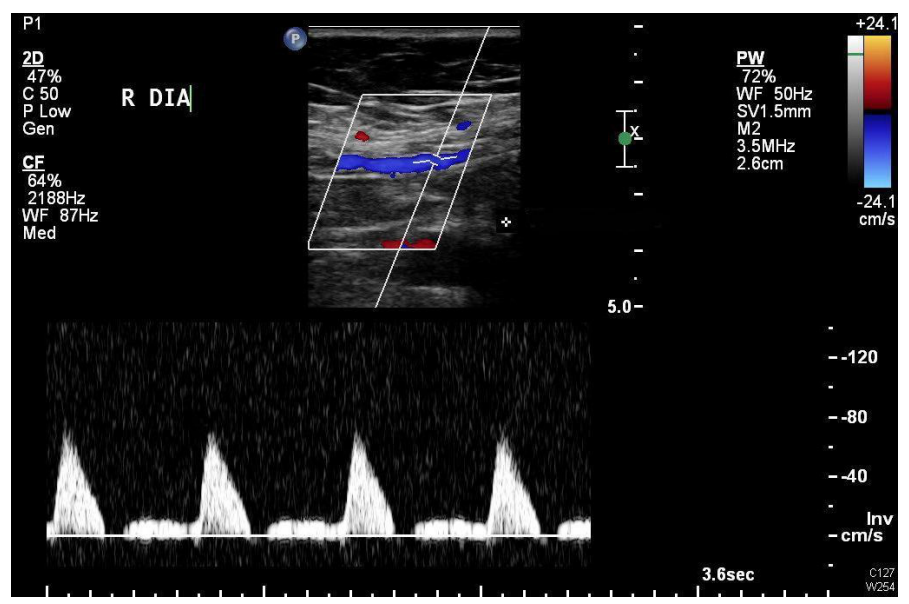

Figure 1: Anonymised Doppler velocimetry of the right deep inferior epigastric artery (DIEA)

Anonymised images of the arterial pulse seen on Doppler scanning were used to generate realistic pulse waves for the inlet boundary. This was carried out using a bespoke algorithm coded in MATLAB R2016b (The MathWorks, Inc.). Here, raw images were converted to grayscale and calibrated manually for time and velocity (Figure 2). Best-fit splines were then overlaid to smooth the data and provide a more accurate representation of the velocity profile (Figure 3). Data points from these smoothed spline curves were exported and used as inlet boundary velocities for the pulsatile simulations.

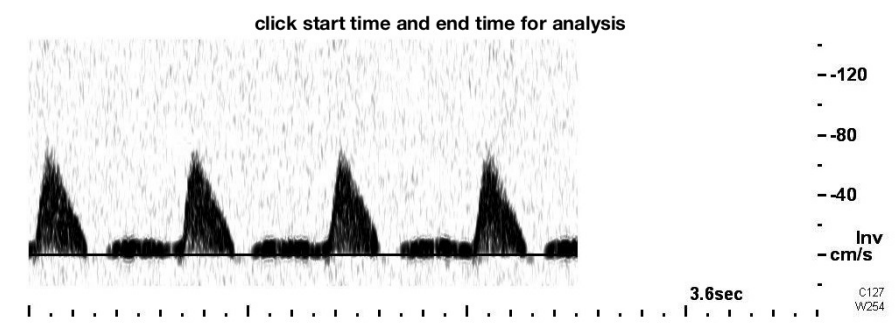

Figure 2: Image calibration using bespoke algorithm coded within MATLAB R2016b (The MathWorks, Inc.)

\subsection{Vessel geometry and meshing}

Two distinct vessel geometries were created using Design Modeler within ANSYS Workbench v16.2. The first being a simple cylinder to represent a pristine vessel for 


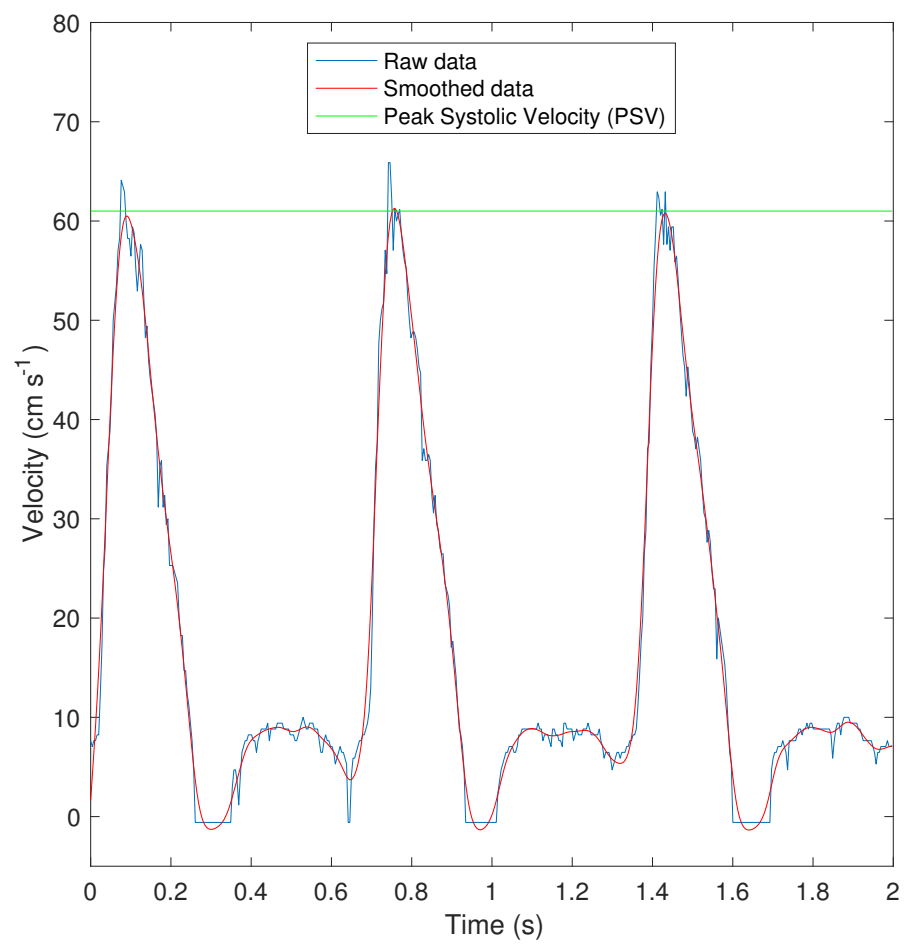

Figure 3: Raw, smoothed and peak systolic velocity (PSV) data displayed in MATLAB R2016b, from calibrated Doppler velocimetry image

verification purposes and the second consisting of a series of circumferential sutures at its midpoint to represent a realistic sutured microarterial anastomosis. Both vessels were $2.5 \mathrm{~mm}$ in diameter and $20 \mathrm{~mm}$ in length. Microvascular sutures were formed in a similar way to our previous study [27] using 8 sutures of $0.04 \mathrm{~mm}$ diameter material to simulate 9/0 Ethilon microsutures (Ethicon, Johnson \& Johnson, Somerville, NJ 08876, USA). Each suture was given dimensions for bite width and orientation (suture angle and spacing) on the vessel wall to represent good microsurgical practice [13, 27]. Suture angle is defined as the suture's deviation from parallel to the vessel's longitudinal axis, bite width represents the size of each individual suture, and spacing refers to the distance between sutures.

Both vessel geometries then underwent a meshing routine consisting of a refinement protocol at the vessel edges and walls in order to best visualise internal flows and surface phenomena. Meshes comprising both tetrahedral and hexahedral elements were created within ANSYS Meshing v16.2. A steady-state mesh independence study was performed with respect to both velocity and SSR whereby mesh densities ranged from $\sim 64,000$ to $\sim 650,000$ cells for the pristine vessel, and from $\sim 1,536,000$ to $\sim 4,300,000$ cells for the sutured vessel. Meshes consisting of at least $\sim 500,000$ and $\sim 3,800,000$ cells were found to produce mesh independent results for the pristine and sutured meshes respectively (Table 1). Here, results were deemed mesh independent when values for velocity and SSR remained constant despite further increases in mesh density, and also were within $1 \%$ of the values seen in analytic solutions for pristine vessels. Figure 4 demonstrates the surface meshes for the sutured geometry. 


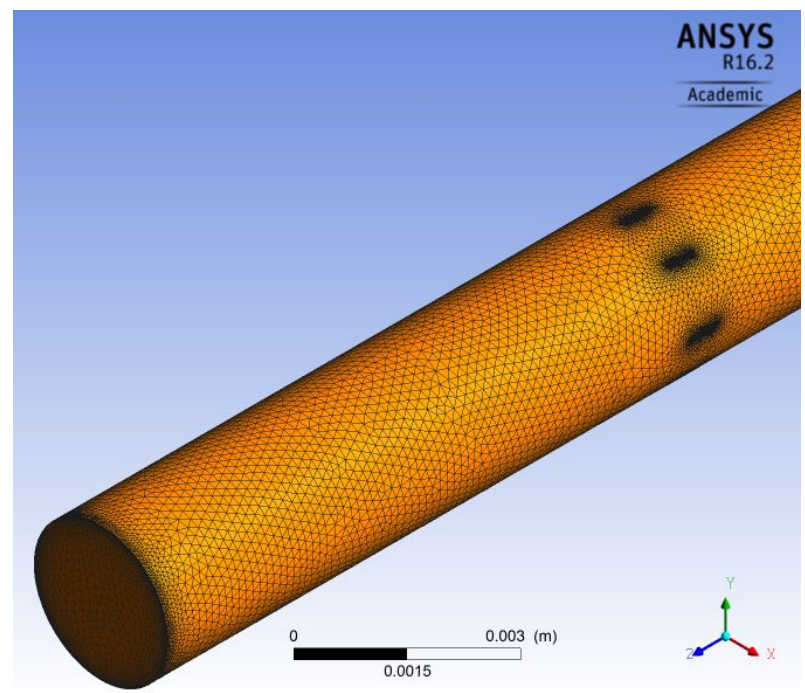

(a) Expanded view of sutured vessel mesh

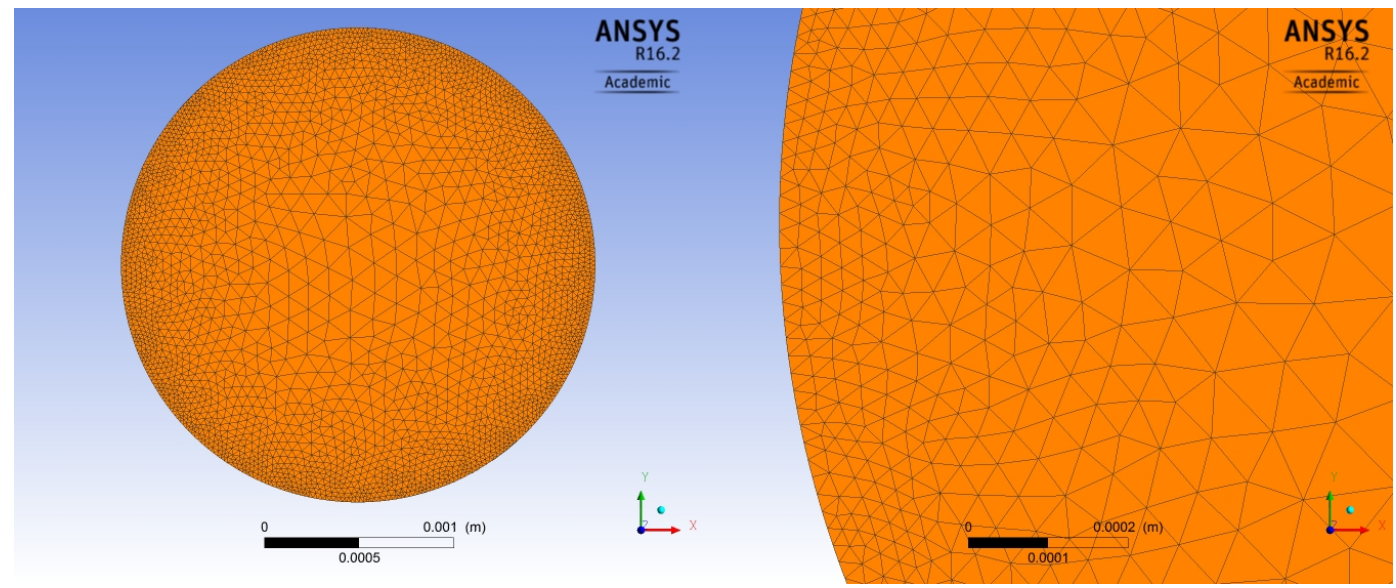

(b) Magnified views of mesh at the inlet detailing element size and configuration. (left) showing the whole inlet and (right) demonstrating refinement at the edge

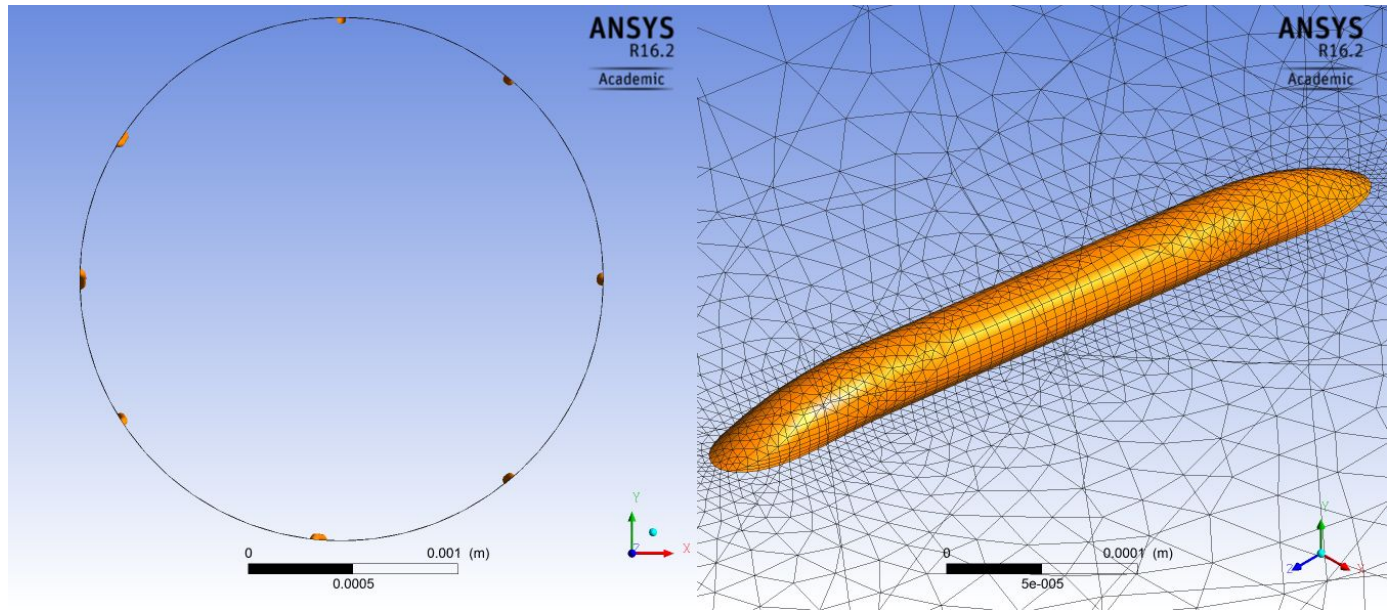

(c) Expanded view to demonstrate suture positions (left) and magnified view (right) detailing mesh on an individual suture

Figure 4: Sutured vessel meshes displaying suture positions and mesh characteristics 


\begin{tabular}{|c|c|c|c|}
\hline Geometry & $\begin{array}{c}\text { No. of elements } \\
\left(\times \mathbf{1 0}^{\mathbf{3}}\right)\end{array}$ & $\begin{array}{c}\boldsymbol{v}_{\mathbf{m a x}} \\
\left(\mathbf{m s}^{\mathbf{- 1}}\right)\end{array}$ & $\begin{array}{c}\mathbf{S S R}_{\max } \\
\left(\mathbf{s}^{\mathbf{- 1}}\right)\end{array}$ \\
\hline \multirow{4}{*}{ Pristine } & 64 & 0.619 & 938 \\
\cline { 2 - 4 } & 126 & 0.619 & 979 \\
\cline { 2 - 4 } & 373 & 0.619 & 981 \\
\cline { 2 - 4 } & 503 & 0.620 & 988 \\
\hline \multirow{4}{*}{ Sutured } & 650 & 0.620 & 988 \\
\cline { 2 - 4 } & 1536 & 0.619 & 2034 \\
\cline { 2 - 4 } & 2514 & 0.619 & 2098 \\
\cline { 2 - 4 } & 3621 & 0.619 & 2114 \\
\cline { 2 - 4 } & 3801 & 0.620 & 2120 \\
\hline
\end{tabular}

Table 1: Mesh dependence study results in steady-state simulations using a parabolic inlet velocity profile $\left(v_{\max }\right)$ of $0.62 \mathrm{~ms}^{-1}$

\subsection{Boundary conditions}

Blood was simulated as a Newtonian fluid with density $(\rho)$ of $1060 \mathrm{~kg} \mathrm{~m}^{-3}$ and dynamic viscosity $(\mu)$ of $3.5 \mathrm{mPa}$ s in keeping with existing literature $[26,27,30,43,44$, $45]$, and was simulated using a laminar solver due to the low Reynolds number $(R e \approx$ $470)$ at peak velocity. The vessel wall was non-compliant and given no-slip conditions for this simulation. All simulations were performed using ANSYS-CFX v16.2.

\subsubsection{Inlet and outlet}

Inlet and outlet boundary conditions were applied in all models. For the simplest steady-state simulations a parabolic velocity profile with a maximum velocity $\left(v_{\max }\right)$ of $0.62 \mathrm{~ms}^{-1}$ was given at the inlet and a relative pressure of zero prescribed at the outlet. This value for velocity was taken from the PSV of the measured arterial waveform. The combination of a velocity inlet and relative pressure outlet condition are recommended by ANSYS as robust for simulating internal flow within ducts [46].

For transient simulations, inlet properties were specified as a rudimentary pulse in the first instance by way of a sine wave of frequency $(f) 1.5 \mathrm{~Hz}$, representing a heart rate of 90 beats per minute, maximum velocity $0.62 \mathrm{~ms}^{-1}$ and minimum velocity of 0 $\mathrm{ms}^{-1}$, as taken from the Doppler ultrasound measurements. The expression posed at the inlet was:

$$
v(t, r)=\frac{v_{\max }}{2}(1+\sin (\omega t))\left(1-\left(\frac{r}{R_{i}}\right)^{2}\right)
$$

where, $v$ is the velocity, $\omega$ denotes the radial oscillating frequency, $t$ is time, $r$ is the spatial radial coordinate, and $R_{i}$ is the inner (lumenal) radius of the vessel.

In the case of realistic pulse simulations, inlet velocities were governed by calculated values of the measured pulse waveform and subsequent smoothed spline curve (Figure 3) with peak flow velocity being approximately $0.62 \mathrm{~ms}^{-1}$. In both transient cases, a parabolic Poiseuille inlet was specified, denoted by the right-hand term in equation (1), and a relative pressure of zero was again set at the outlet. All transient simulations were performed over 2 seconds with time-steps of 0.01 seconds. 


\subsection{Simulation strategy and post-processing}

Verification was performed using both steady-state and sinusoidally oscillating pressure driven flows in a cylindrical pipe, essentially classical problems from fluid mechanics $[47,48]$. The problem solved was the unidirectional transient Navier-Stokes equation:

$$
\rho \frac{\partial v}{\partial t}+\frac{\partial p}{\partial z}=\frac{\mu}{r} \frac{\partial}{\partial r}\left(r \frac{\partial v}{\partial r}\right)
$$

where $p$ is the pressure driving the flow, and $v$ denotes blood flow velocity through the vessel.

For verification purposes all pristine simulations were evaluated for velocity and pressure drop, exploiting symmetry in $y$ - $z$ plane. Shear strain rate (SSR) was also evaluated in an attempt to visualise foci of potential activation of platelets and subsequent thrombus formation. In particular the shear gradient magnitude, defined as the change of SSR along an individual suture (equation 3), was evaluated by the software to gain an appreciation of the propensity to form thrombi. SSR gradient ( $\mathrm{SSR}_{\text {grad }}$ ) was defined as:

$$
\mathrm{SSR}_{\text {grad }}=\frac{\left(\dot{\gamma}_{\text {top }}-\dot{\gamma}_{\text {trail }}\right)}{\Delta z}
$$

where $\dot{\gamma}_{\text {top }}$ and $\dot{\gamma}_{\text {trail }}$ represent the SSR at the top and trailing edges of the suture, and $\Delta z$ is the distance between the two monitor points.

\section{Results}

This section begins with the simplest pristine vessel in a steady-state, moves on to transient simulations, and concludes with a realistic pulse in a sutured microarterial anastomosis. Verification using analytic solutions is provided where appropriate, and comparisons are drawn between these and simulation results, to determine the influence of pulsatile flow on local haemodynamics. Where CFD simulations were used, all results were independent of mesh density.

\subsection{Pristine vessel verification - steady-state and sinusoidal flow}

A pristine case has been used as a benchmark in both steady-state and transient simulations. The steady-state vessel simulation at PSV demonstrated a parabolic velocity profile throughout, and a uniform pressure drop of approximately $120 \mathrm{~Pa}$, equivalent to almost $1 \mathrm{mmHg}$, along the $20 \mathrm{~mm}$ section. Figure 5 shows velocity profile plots at intervals along the pristine vessel's length for both steady-state and sinusoidal inlet conditions. In keeping with imposed inlet boundary conditions of a continuous PSV, the maximum central velocity was recorded as $0.62 \mathrm{~ms}^{-1}$ in the steady-state case. Values for $\mathrm{SSR}_{\text {max }}$ at the inner (lumenal) radius of the vessel wall $\left(\dot{\gamma}\left(R_{i}\right)\right)$ were $988 \mathrm{~s}^{-1}$ (Figure 6). This value of $\mathrm{SSR}_{\max }$ is in agreement with previous work [27] and is also within $1 \%$ of the analytic solution for Poiseuille pipe flow for a circular duct of comparable size from White [47] $\left(992 \mathrm{~s}^{-1}\right)$ also shown in Figure 6. The Womersley number was calculated to be 2.11 for these simulations.

The addition of a sinusoidal inlet condition produced analogous velocity and pressure data to the steady-state solution. Figure 5 demonstrates consistency of the velocity parabola in the transient sinusoidal model when compared to the steady-state benchmark. 
$\mathrm{SSR}_{\max }$ was calculated at $997 \mathrm{~s}^{-1}$ using CFD which is within approximately $1 \%$ of the maximum value in the steady simulation $\left(988 \mathrm{~s}^{-1}\right)$, depicted in Figure 6. In sinusoidal flows, comparison of the CFD SSR $\max$ value $\left(997 \mathrm{~s}^{-1}\right)$ with the analytic solution (1014 $\mathrm{s}^{-1}$ ) gives an error of $1.6 \%$. This, in conjunction with an error of less than $1 \%$ between steady-state solutions, indicates computational simulations are in agreement with each other and with analytic solutions.

(a)

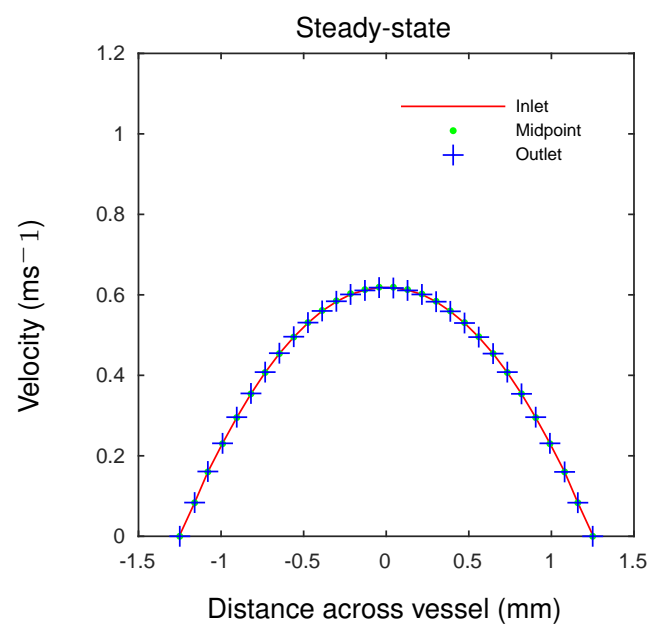

(c)

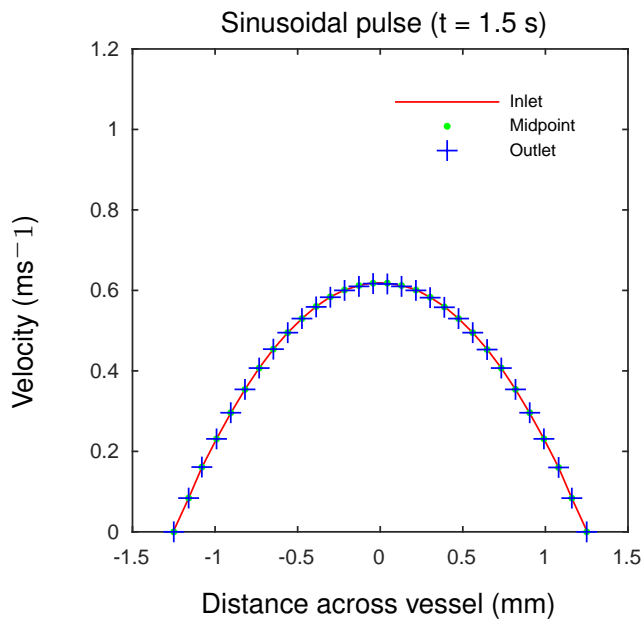

(b)

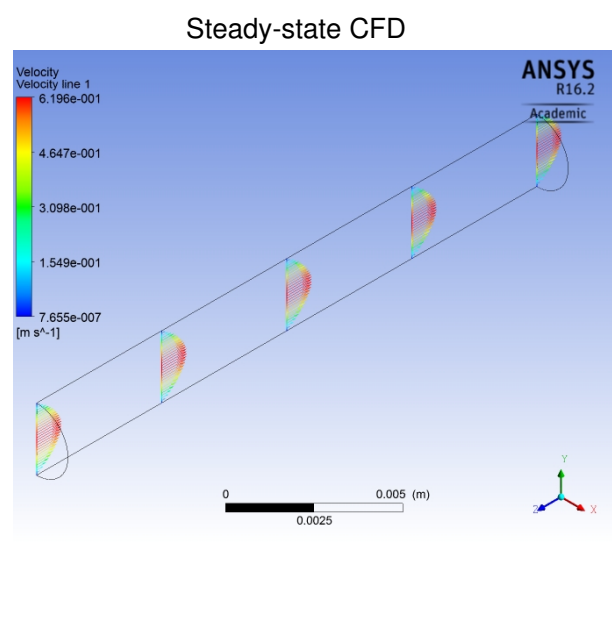

(d)

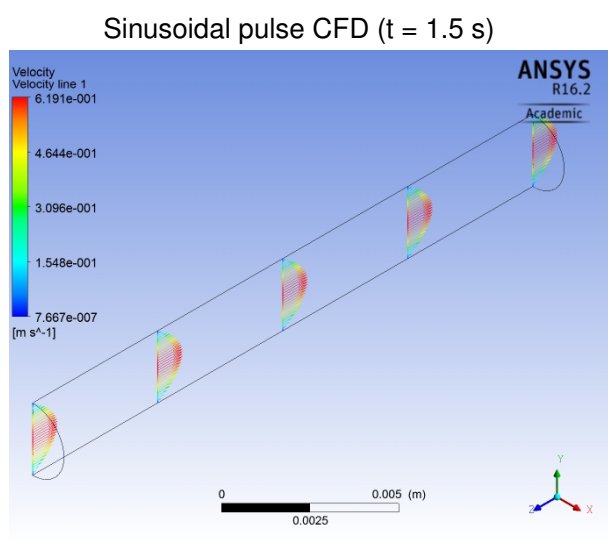

Figure 5: Velocity profiles plotted across the diameter of the pristine vessel at its inlet, midpoint and outlet. Figures (a) \& (b) demonstrate the steady-state case, and figures (c) \& (d) represent the sinusoidal case taken at $\mathrm{t}=1.5 \mathrm{~s}$ i.e. at $v_{\max }$. 

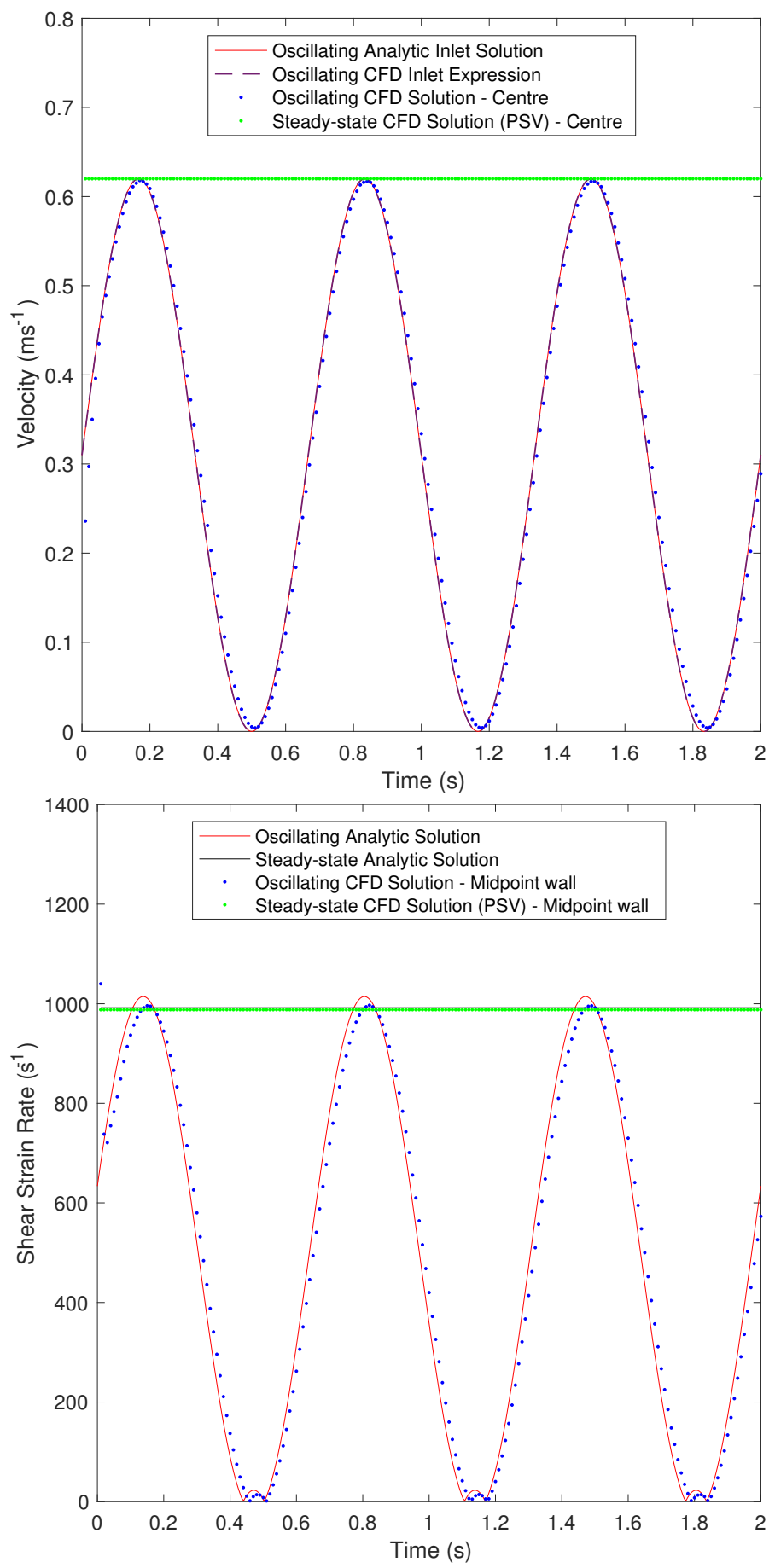

Figure 6: Pristine vessel verification - analytic and CFD sinusoidal pulse, and steady-state (PSV) CFD data (Velocity (top) \& SSR (bottom) where $\mathrm{SSR}_{\max }$ is plotted for steady-state, and $\mathrm{SSR}_{\mathrm{t}}$ for transient cases).

Also for comparative purposes a transient analytic pressure driven model is shown in Figure 6. This is derived in a similar way to that by Sexl [48] and White [49], by differentiation of the solution to equation (2), and incorporating the steady and transient 
aspects, thus:

$$
v(r, t)=\frac{C_{0}}{4 \mu}\left(r^{2}-R_{i}^{2}\right)+\Re\left(\frac{C_{\max }}{i \omega \rho}\left[1-\frac{J_{0}\left(i^{\frac{3}{2}} \sqrt{\frac{\rho \omega}{\mu}} r\right)}{J_{0}\left(i^{\frac{3}{2}} \sqrt{\frac{\rho \omega}{\mu}} R_{i}\right)}\right] e^{i(\omega t+\varphi)}\right)
$$

where $J_{0}$ is a Bessel function of the first kind, $\omega$ is the radial oscillating frequency, and $\varphi$ is a phase angle used to match the pressure driven solution to the inlet velocity driven solution, defined as:

$$
\varphi=-\frac{1}{\omega} \operatorname{Arg}\left(1-J_{0}\left(i^{\frac{3}{2}} \sqrt{\frac{\rho \omega}{\mu}} R_{i}\right)^{-1}\right) .
$$

Differentiation of equation (4) with respect to $r$ renders the SSR $(\dot{\gamma}(r, t))$ where the pressure gradient is:

$$
\frac{d p}{d z}=C_{0}+C_{\max } \cos (\omega(t+\varphi))
$$

where $C_{0}=-2 v_{\max } \mu / R_{i}^{2}$ represents the time-independent component, and $C_{\max }$ represents the time-dependent component, defined as:

$$
C_{\max }=\frac{\omega \rho v_{\max }}{2\left|1-J_{0}\left(i^{\frac{3}{2}} \sqrt{\frac{\rho \omega}{\mu}} R_{i}\right)^{-1}\right|} .
$$

Good agreement (error of $\leq 1.6 \%$ ) is demonstrated between the analytic transient solution (equation (4)), the prescribed velocity inlet expression (equation (1)), and the ANSYS-CFX data for both velocity and SSR (Figure 6), thereby verifying the ANSYSCFX simulation methods used herein.

\subsection{Pristine vessel - realistic pulse}

Realistic velocities from the Doppler velocimetry (Figure 3) were specified at the inlet in this simulation, with the remainder of the parameters and geometry remaining constant. This imposed a fully developed parabolic velocity profile in systole $\left(v_{\max }=\right.$ $\left.0.62 \mathrm{~ms}^{-1}\right)$. Reciprocal changes in the pressure drop along the vessel length were seen, ranging from approximately $182 \mathrm{~Pa}$ in systole to just $12 \mathrm{~Pa}$ in diastole. With respect to $\mathrm{SSR}_{\max }$, a value of $1090 \mathrm{~s}^{-1}$ was calculated at $v_{\max }$ which is slightly higher than that calculated in the PSV steady-state $\left(988 \mathrm{~s}^{-1}\right)$ and sinusoidal CFD simulations $\left(997 \mathrm{~s}^{-1}\right)$ (Figure 7). 


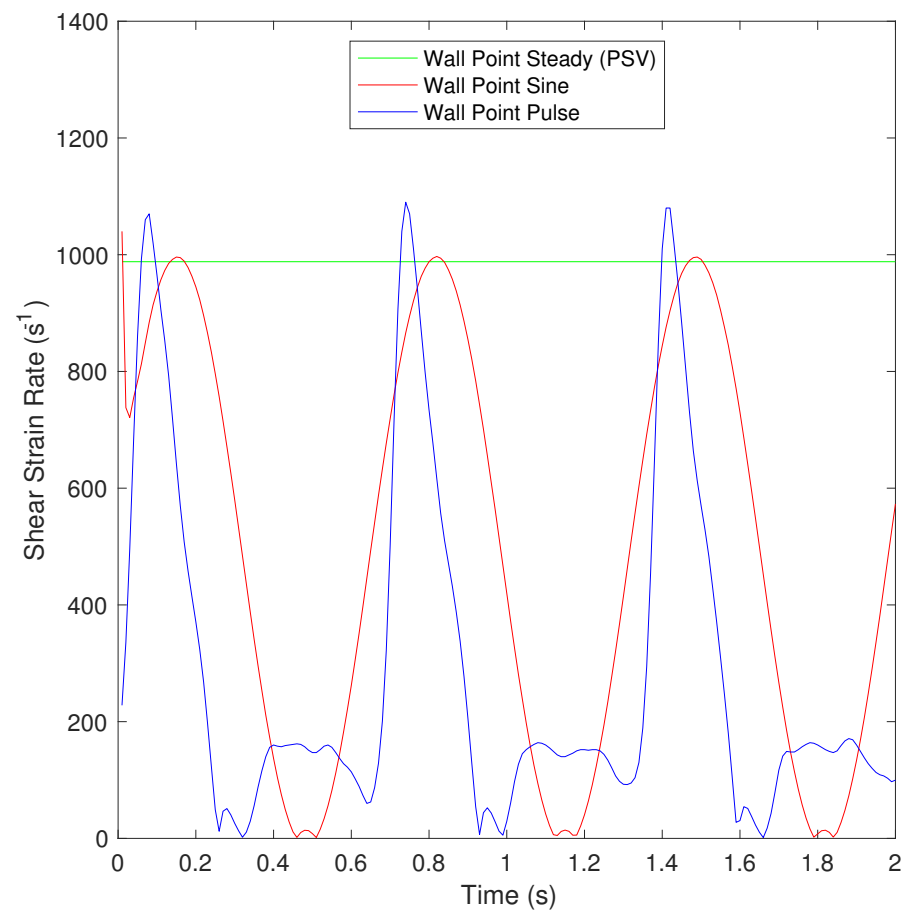

Figure 7: SSR comparison: steady-state (PSV), sinusoidal and realistic pulses showing SSR $_{\max }$ for steady-state, and $\mathrm{SSR}_{\mathrm{t}}$ for transient cases in a pristine vessel.

Videos showing realistic transient flows are available as supplementary online material.

\subsection{Sutured vessel - steady-state and sinusoidal flow}

Sutured simulations featured a consistent geometrical change compared to the pristine vessel representing eight microsurgical sutures with a small degree of variation in length, width and spacing in keeping with good microsurgical practice (Figure 4c). Specific consistent monitor points were used at the top (innermost) and trailing (downstream) edges of two of the eight sutures (Figure 8) in an attempt to ensure uniformity between simulations. These monitor points were anticipated to detect the gradient between high and low shear regions along the suture. Suture positions remained unchanged throughout the study. 


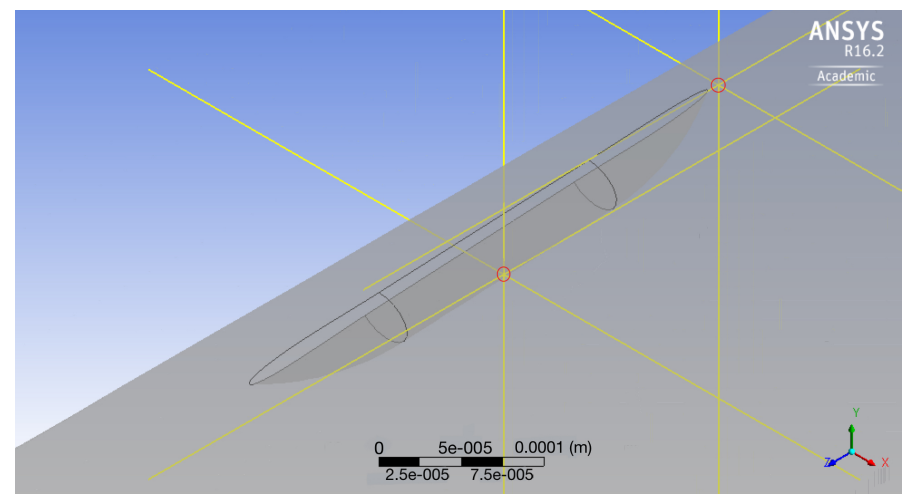

Figure 8: Monitor points (red circles) - sutured geometry

Velocity fields in the central flow were almost identical in both sutured and pristine steady-state simulations, as was the pressure drop along the vessel. Values for maximum SSR were, however, notably different. At the top of the sutures, $\mathrm{SSR}_{\max }$ for both Suture $1\left(2120 \mathrm{~s}^{-1}\right)$ and Suture $3\left(1779 \mathrm{~s}^{-1}\right)$ was approximately double that found in the pristine model $\left(988 \mathrm{~s}^{-1}\right)$, depicted by the dashed horizontal lines in Figure 10. At the suture's trailing edges, $\mathrm{SSR}_{\max }$ was conversely found to be less than that found in the pristine vessel, with values of $647 \mathrm{~s}^{-1}$ and $825 \mathrm{~s}^{-1}$ representing a decrease of $\sim 34 \%$ and $\sim$ $17 \%$ for Sutures 1 and 3 respectively. The suture trailing edge values are lower than the analytic value of $992 \mathrm{~s}^{-1}$ by the same magnitude, to within $<1 \%$. These findings were consistent in each simulation and are reflected in the ANSYS-CFX contour plots (Figure 9). Here, a generalised increase of $\sim 60 \%$ in $\mathrm{SSR}_{\max }$ is seen in the anastomotic region (approximately $\left.1600 \mathrm{~s}^{-1}\right)$ compared to the baseline of a pristine vessel $\left(988 \mathrm{~s}^{-1}\right)$. This varies according to suture spacing and orientation, evidenced by the differing values between Sutures 1 and 3, and is supported by findings of previous work [27]. Figure 9 also depicts a magnified view of a single suture SSR contour plot with additional velocity streamlines. High shear rates are seen on the suture's top edge, with lower shear values at its trailing edge. It is also possible to visualise slower flow where the velocity arrows are condensed, and faster flow where the streamlines are more widely distributed, as a result of the angle of suture placement - albeit small. 


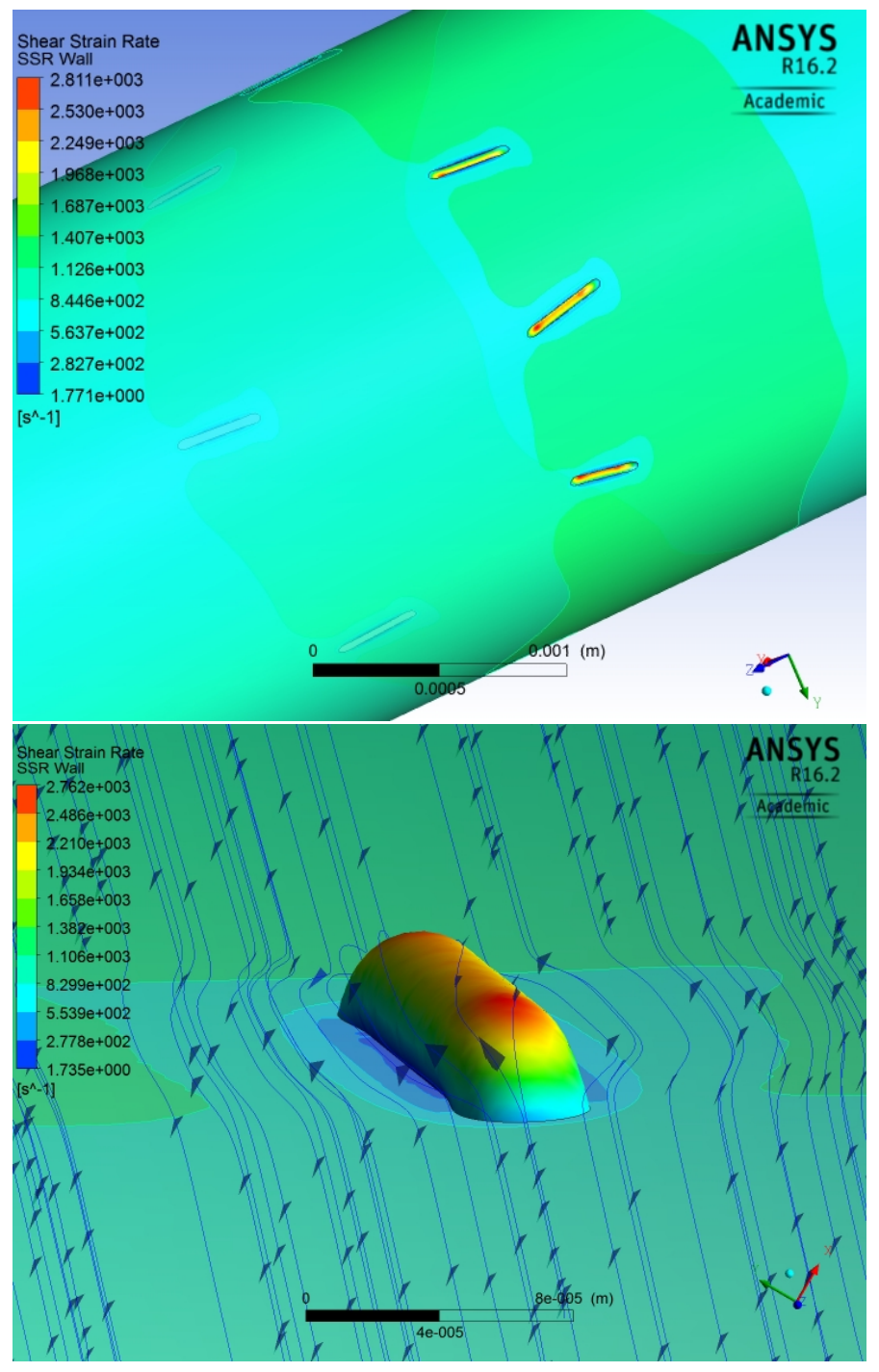

Figure 9: Sutured SSR Contours $\left(\mathrm{s}^{-1}\right)$ : (top) whole vessel showing three sutures, (bottom) magnified view of a single suture with velocity streamlines and directional arrows. These images are from steadystate simulations i.e. at $v_{\max }=0.62 \mathrm{~ms}^{-1}$.

Incorporation of a sinusoidal pulse wave to the sutured simulation produced a $\mathrm{SSR}_{\max }$ of slightly greater magnitude (by 1-2\%) than the PSV steady-state at each monitor point. This was more pronounced at the suture's top than the trailing edge in all cases, as can be seen in Figure 10. Again, a similar pattern was seen using a sinusoidal inlet as for the steady-state simulation regarding the suture's trailing edges, where values of $\mathrm{SSR}_{\max }$ for Sutures 1 and 3 were $\sim 36 \%$ and $\sim 19 \%$ less than the sinusoidal analytic solution, respectively. 

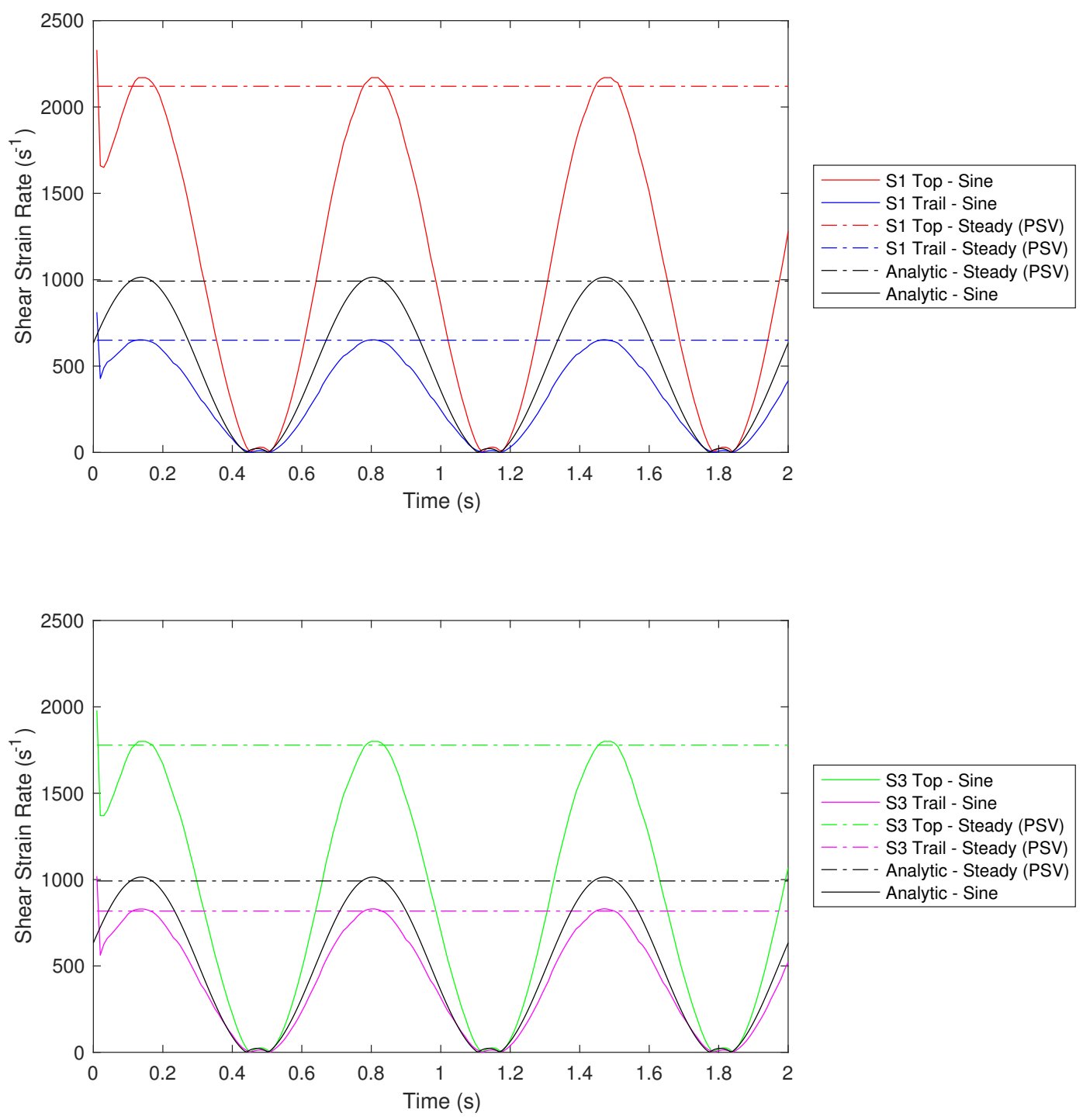

Figure 10: SSR comparison: steady-state (PSV) and sinusoidal pulse in a sutured vessel for Suture 1 (top) and Suture 3 (bottom). Monitor points as per Figure (8) for each sutured geometry. SSR max $_{\text {is }}$ plotted for steady-state and $\mathrm{SSR}_{\mathrm{t}}$ for transient cases. Analytic solutions are plotted in black, whilst CFD numerical results are plotted in colour.

\subsection{Sutured vessel - steady-state and realistic pulse}

These sutured simulations featured identical geometries, with only inlet velocity changing from the steady-state PSV value of $0.62 \mathrm{~ms}^{-1}$, to the realistic pulsatile velocity (Figure 3), as in the pristine model. Here, the SSR is observed to be greatest at the top of the sutures and lowest at their trailing edges at each time-step (Figure 11), as for the steady-state and sinusoidal simulations described above. This is a consistent finding for each suture despite its position i.e. Suture 1 (S1) and Suture 3 (S3) both demonstrate the same relationship, yet to different extents owing to the subtle differences in their angle, bite width and spacing.

Maximum and minimum SSRs in the realistic pulse solution are consistently greater 
than the PSV steady-state solution, although this is more pronounced at the top of the suture rather than it's trailing edge. This can be seen clearly in Figure 11, with pulsatile flow represented by solid lines, and steady-state depicted with dashed lines. It is therefore implied that a steady-state simulation systematically underestimates $\mathrm{SSR}_{\max }$ in these geometries.

Direct analysis of the maximum SSRs obtained at corresponding monitor points (Table 2) has demonstrated a factor of approximately 1.18-1.19 can be used to calculate $\mathrm{SSR}_{\max }$ seen at the suture tops in a realistic pulse from that seen in a steady-state solution at PSV. Incidentally, this factor is significantly increased when taking into account time-averaged flow rather than peak flow rates for the steady-state sutured simulation. Here, numerical integration can be used to analyse the realistic pulse waveform, rendering an equivalent time-averaged steady-state $v_{\max }$ of $0.17 \mathrm{~ms}^{-1}$, and thereby a factor of approximately 4.10 to predict maximum SSRs in these simulations.

\begin{tabular}{|c|c|c|c|}
\hline Suture & $\begin{array}{c}\text { Steady }(\mathbf{P S V}) \\
\mathbf{S S R}_{\max }\left(\mathbf{s}^{\mathbf{- 1}}\right)\end{array}$ & $\begin{array}{c}\text { Pulse } \\
\mathbf{S S R}_{\max }\left(\mathbf{s}^{-\mathbf{1}}\right)\end{array}$ & $\begin{array}{c}\text { Pulse } \\
\text { Steady (PSV) }\end{array}$ \\
\hline S1 Top & 2120 & 2530 & 1.19 \\
\hline S1 Trail & 647 & 717 & 1.11 \\
\hline S3 Top & 1779 & 2090 & 1.18 \\
\hline S3 Trail & 825 & 932 & 1.13 \\
\hline
\end{tabular}

Table 2: Steady-state (PSV) and realistic pulse SSR $_{\max }$ comparison for a sutured anastomosis. Factors to predict the influence of realistic pulsatile flow are shown in the right column 

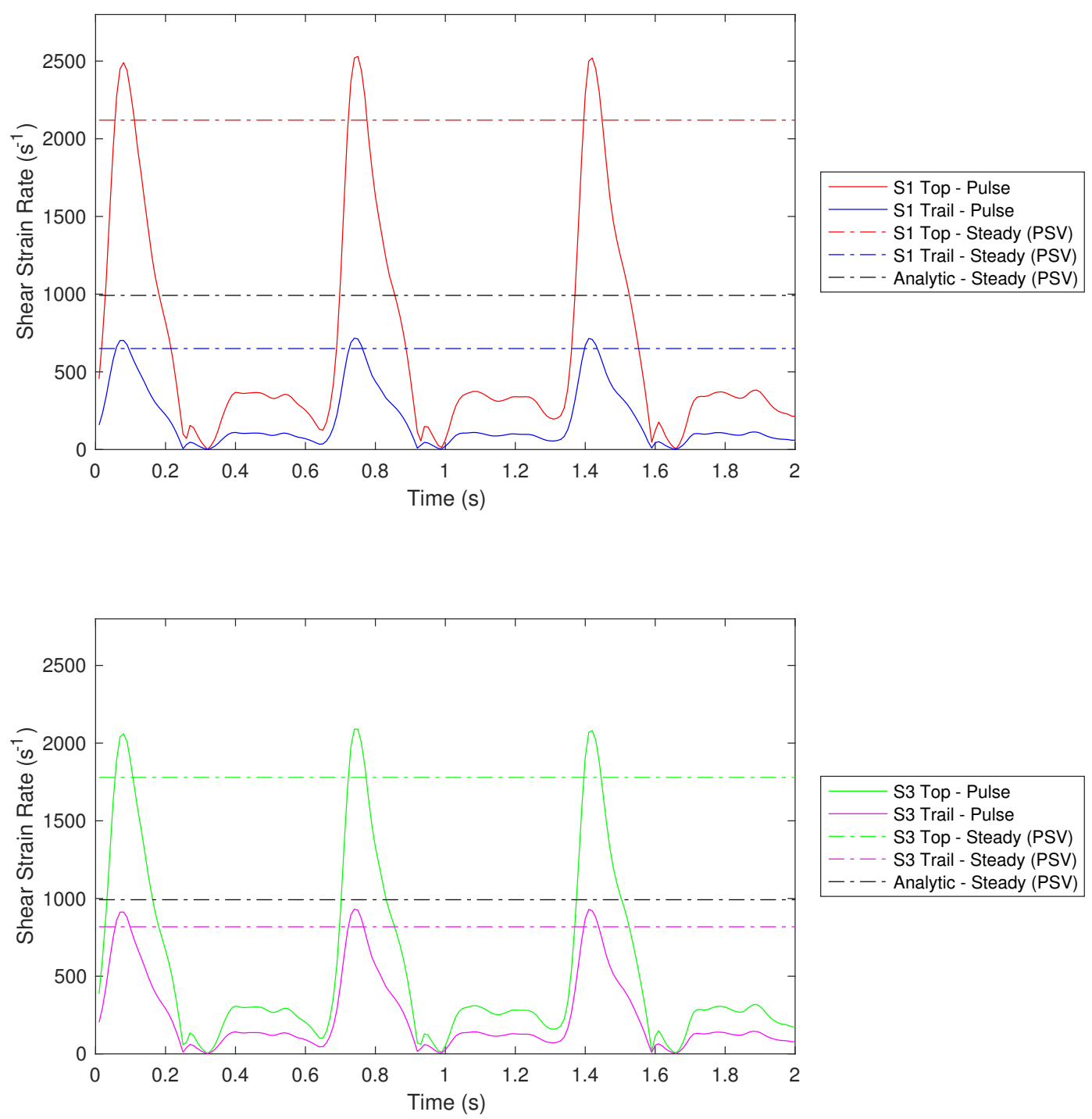

Figure 11: SSR comparison: steady-state (PSV) and realistic pulse in a sutured vessel for Suture 1 (top) and Suture 3 (bottom). Monitor points as per Figure (8) for each sutured geometry. SSR $\mathrm{Sax}_{\text {is }}$ plotted for steady-state and $\mathrm{SSR}_{\mathrm{t}}$ for transient cases. Analytic solutions are plotted in black, whilst CFD numerical results are plotted in colour.

Videos showing these realistic transient flows in both pristine and sutured vessels are available as supplementary online material.

\subsection{Realistic pulse - sutured and pristine vessels}

Whilst the above results demonstrate increasing $\mathrm{SSR}_{\max }$ values due to the presence of pulsatile flow, it is important these are viewed in context of SSRs present in a natural pristine vessel. Figure 12 illustrates the $\mathrm{SSR}_{\max }$ values seen in both cases under realistic pulsatile flow. 


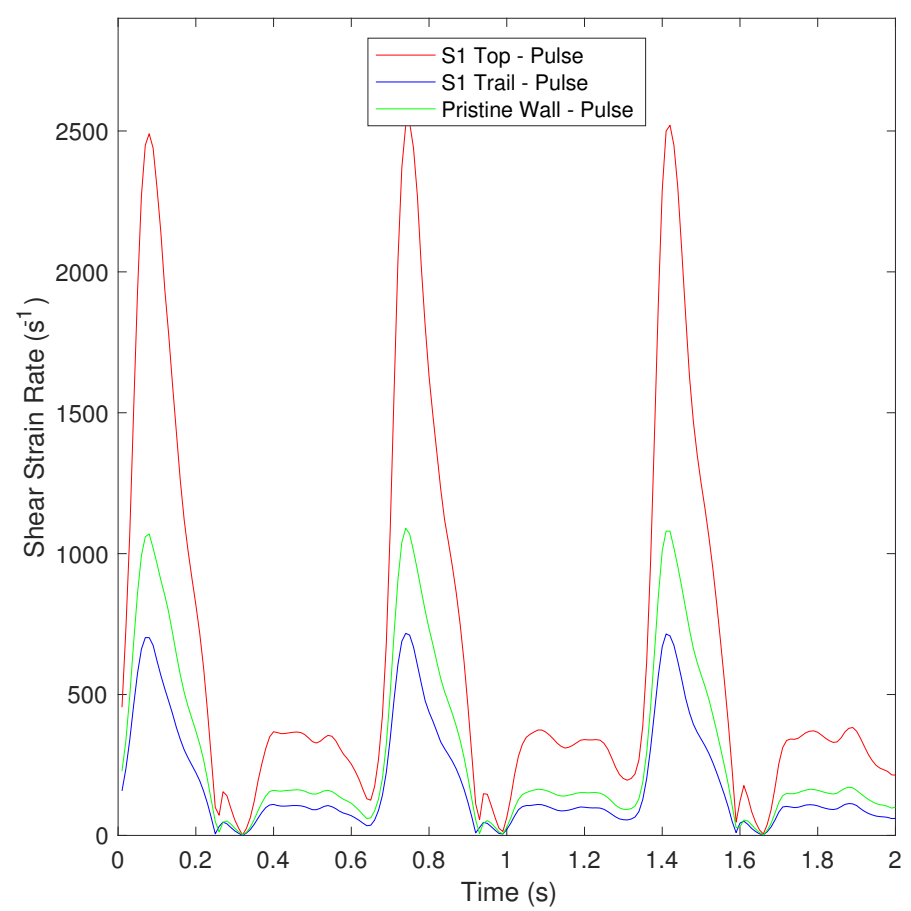

Figure 12: $\mathrm{SSR}_{\max }$ comparison of realistic pulse in sutured and pristine vessels using CFD. Monitor points as per Figure (8) for sutured geometry, and a consistent wall monitor point at the vessel midpoint for pristine geometry

At the peak of systole $\left(v_{\max }\right)$ the maximum SSR in the sutured anastomosis is 2520 $\mathrm{s}^{-1}$, and is seen at the suture's top. This value is $\sim 2.5$ times greater than that seen in a pristine vessel $\left(988 \mathrm{~s}^{-1}\right)$. Again at the peak of systole, the minimum SSR in sutured anastomosis is $717 \mathrm{~s}^{-1}$, and can be found at the suture's trailing edge. The value here is $\sim 30 \%$ less than that seen in a pristine vessel at the same time $\left(988 \mathrm{~s}^{-1}\right)$.

Moving forward from the calculations in Table 2, shear gradients have been predicted along sutures $1 \& 3$, and are shown in Figure 13. This specifically demonstrates the change in shear seen along a single suture using the measured arterial pulse. Magnitudes of these gradients are very high at the peak of systole, at around $7.9 \times 10^{6} \mathrm{~m}^{-1} \mathrm{~s}^{-1}$, which may be associated with activation of platelets and formation of aggregates. Although it is unclear at precisely what gradient platelet activation would occur, it has been demonstrated that the magnitude and spatial distribution of shear micro-gradients directly influences platelet aggregate size [24]. 


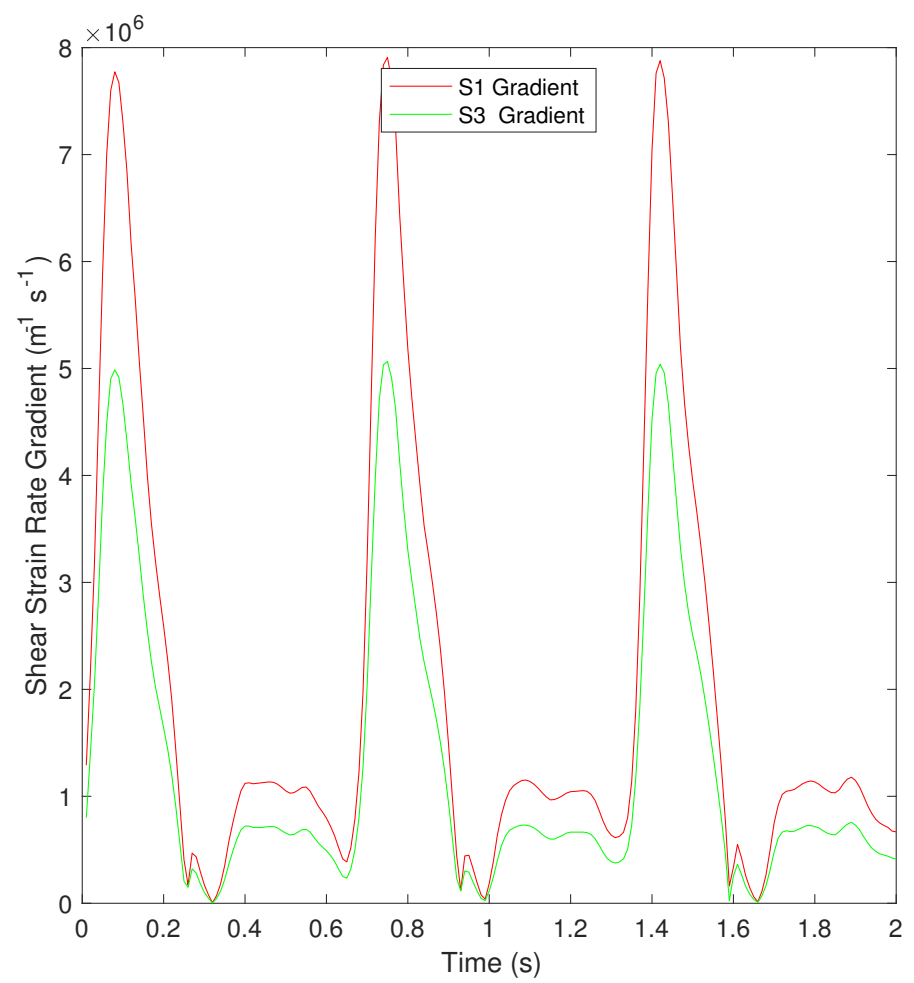

Figure 13: SSR gradients in sutured microarterial anastomosis with realistic pulsatile flow using CFD. Monitor points as per Figure (8) for both Suture 1 (S1) and Suture 3 (S3)

\subsection{Statistical analysis}

Statistical analysis has been performed using MATLAB R2016b (The MathWorks, Inc.). Student's $t$-tests were carried out to evaluate the difference between pulsatile and steady-state (PSV) values of SSR in the sutured and pristine anastomoses. Ten separate calculations of $\mathrm{SSR}_{\max }$ were performed for each simulation. Top-trailing pair SSR values were obtained from the same suture at different time points corresponding to PSV in the transient case. SSR values were taken from the same locations in the steady-state case. Ten sets of values were sampled from areas in the immediate vicinity of the standardised monitor points such that the groups of data were equivalent to enable comparison. The null hypothesis being that pulsatile flow does not influence SSR when compared to steady-state. The $t$-critical value for this analysis was 2.83 at the $99 \%$ confidence level.

In each analysis the null hypothesis was rejected $\left(t>60, p \approx 5.06^{-14}\right)$ thereby confirming observations from Figure 11 that pulsatile flow significantly increases SSR $_{\max }$ compared to a steady-state simulation at PSV. The effect is more pronounced in a sutured anastomosis than a pristine vessel, which is in keeping with the values seen in Figures $7 \& 11$.

\section{Discussion}

This paper has investigated the effect of pulsatile flow on local haemodynamics in microvascular anastomoses, when compared to a steady-state approximation at peak 
systolic velocity. Computational simulations were designed to evaluate flow properties firstly in pristine vessels, and subsequently in geometries representing hand-sutured microarterial anastomoses, each with steady-state and pulsatile components. This permitted direct comparison and verification using analytic steady and transient solutions. Direct experimental measurements of such parameters would present great challenges in vivo.

Existing methods for incorporating pulsatile flow in vessel simulations include, but are not limited to, simple sine waves with time-averaged velocities [34]; values from physiology texts $[41,45]$; data from Doppler ultrasound [50, 51] or Phase-Contrast MRI [52]; and experimental values using transit-time ultrasound in rat vessels [35] or indeed cadaveric aortas [53]. Of these, only studies by Rickard et al. [35] and Al-Sukhun et al. [34] have explored microvascular transient flows, but neither have used pulsatile measurements from humans or specifically investigated sutured anastomoses. Also, neither study directly compared their results to analytic solutions for steady, or pulsatile flows. Whilst not specifically investigating vessels of the same calibre or flow rate as this work, there have been a range of other published studies using CFD to evaluate time-dependent blood flows in small vessels. These include transient fluid-structure interaction (FSI) simulations carried out in retinal arteries over varying perfusion pressures [38]; investigating the influence of blood viscosity in a small $(0.6 \mathrm{~mm})$ bifurcated artery using a non-Newtonian rheological function [40]; and exploring the influence of varying velocity on platelet activation and thrombus formation in arterioles [42]. The latter, along with methods discussed by Perdikaris et al. [39] in their review of simulation in cerebral blood flows, may be applicable to microanastomotic simulation, particularly regarding the dissipative particle dynamics (DPD) approach for predicting platelet activation and thrombus growth. These techniques could be incorporated in future simulations, but are beyond the scope of the current study.

Arterial thrombus formation is directly related to shear strain rate. As arterial SSR increases, a corresponding increase in platelet deposition is seen $[6,54,55,56]$. More platelets flow preferentially near the lumenal wall, so-called margination. Here they become activated, resulting in increased adhesion and cohesion, subsequently increasing thrombus formation $[6,54,56,57,58]$. Placing an absolute value on the SSR initiating these processes is difficult, as they are dependent on multiple factors including vessel size, flow rate, and vessel geometry i.e. presence of stenoses or anomalies in the wall. Normal, physiological shear rates as low as $10 \mathrm{~s}^{-1}$ are seen in venous flows, with rates of around $500 \mathrm{~s}^{-1}$ and $350 \mathrm{~s}^{-1}$ seen in coronary and external carotid arteries respectively, with diameters of approximately $3.5 \mathrm{~mm}$ [7]. Interpolation of data from Figure 2 of [7] renders a physiological SSR of $\sim 900 \mathrm{~s}^{-1}$ for vessels of $2.5 \mathrm{~mm}$ diameter, which compares favourably to the analytic and simulated values obtained herein for pristine vessels.

Pathological conditions such as an arterial stenosis can lead to remarkably high SSR values, with very severe stenosis recording over $32000 \mathrm{~s}^{-1}$ in laboratory based studies $[55,56,59]$. In work by the same groups however, SSRs of $2600 \mathrm{~s}^{-1}$ were seen in diffuse atherosclerosis and moderate arterial stenosis, with the latter demonstrating thrombus formation at the stenosis apex $[55,56,59]$. It could therefore be inferred that the $\mathrm{SSR}_{\max }$ values seen in our pulsatile sutured anastomosis $\left(\sim 2530 \mathrm{~s}^{-1}\right)$ resemble those seen in moderate arterial stenoses, and as such have more potential to develop thrombus than a pristine vessel. Increasing shear rates are also directly related to increasing thrombus 
growth rates, with SSR values of 2000-5000 $\mathrm{s}^{-1}$ producing the greatest thrombus growth in work by Bark and $\mathrm{Ku}$ [58]. The values obtained here can also in some way be compared to those seen in studies evaluating cardiac stent placement and design using CFD [60, 61, 62, 63]. In particular, work by Jiménez [60] demonstrated SSR values ranging from approximately $1600 \mathrm{~s}^{-1}$ to $10000 \mathrm{~s}^{-1}$ in $3 \mathrm{~mm}$ vessels depending on the design specification of a particular intravascular stent. Here, designs were modified by streamlining struts to minimise flow separation and reduce SSRs. Struts producing the lowest SSR values were slim, low profile, and curved - not dissimilar to the microvascular sutures simulated in this study.

It is clear from the data presented that time-resolved simulation of intravascular flows using sinusoidal or realistic pulsatile flow influences the $\mathrm{SSR}_{\max }$ value recorded (Figure 11). This effect is greatest in sutured simulations when compared to a pristine vessel, and more pronounced with realistic pulsatile flow than a sinusoidal approximation. To date, similar findings have not been reported in the body of literature available, indeed our findings are in contrast to those reported by Tenekecioglu [37, 64] when evaluating coronary stent haemodynamics, where no significant difference in endothelial shear rates were seen between steady and pulsatile flows. Similar findings were also reported in a further study evaluating coronary stents [41], although this paper describes timeaveraged values which may influence the conclusion somewhat. Whilst earlier studies performed using steady-state simulations [26, 27, 28] have given insight to the flow properties within microvascular anastomoses, this work has demonstrated that transient flows provide a more accurate picture, particularly when evaluating $\mathrm{SSR}_{\max }$. 


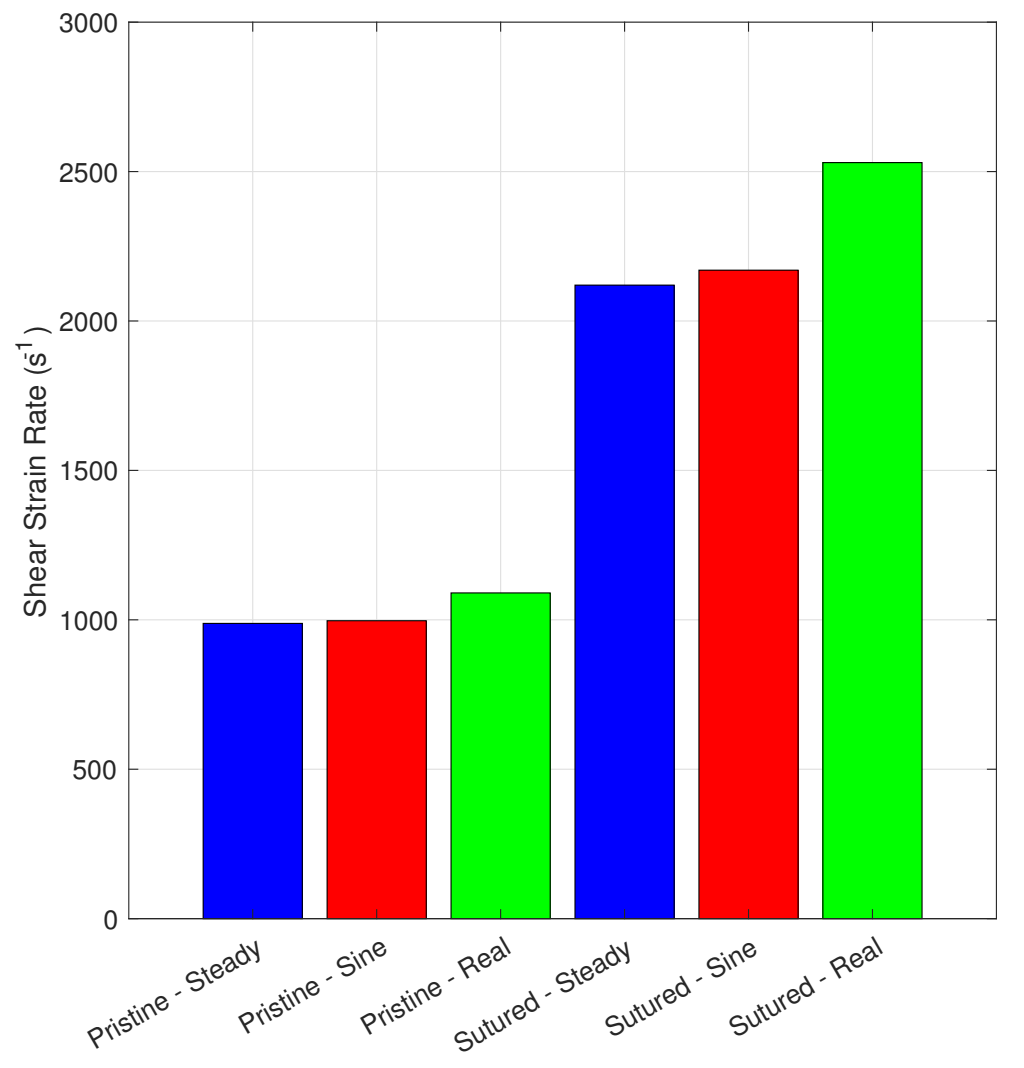

Figure 14: $\mathrm{SSR}_{\max }$ for each CFD simulation recorded at monitor points as per Figure (8). Bar colours are consistent for inlet conditions comparing steady, sinusoidal and realistic pulsatile flows

The extent to which a protuberance within the lumen influences SSR is related to its size, the vessel diameter, and blood flow rate. Shear gradients have been implicated in the formation of thrombus [24], with the rate and size of platelet aggregation correlating directly with shear gradient magnitude and spatial distribution. Interpolating results from Nesbitt et al., very large shear gradients, of the order $\sim 2 \times 10^{10} \mathrm{~m}^{-1} \mathrm{~s}^{-1}$, were seen in severely stenotic microvessels demonstrating maximal shear rates of $\sim 20,000 \mathrm{~s}^{-1}$ at the stenosis apex. These vessels were around $15 \mu \mathrm{m}$ diameter and were subjected to a $90 \%$ stenosis, thereby accounting for the order of magnitude seen. Indeed, platelet aggregation was found not to initiate in the absence of any shear gradients [24]. We quantify this aspect in our simulations by calculating the shear gradients for the given sutures, using a measured arterial waveform (Figure 13), to provide an indication of its potential impact on thrombus formation. Large SSR gradients were observed along sutures in our simulations, with SSR reductions at the trailing edges likely due to deceleration of flow in the immediate downstream vicinity of the suture. This demonstrates similarities, although to a lesser extent, to that described in arterial stenoses [7, 18, 24]. Furthermore, it has been demonstrated that platelets with elevated activation potential have a propensity to gather in downstream regions of deceleration and recirculation which may substantially increase the likelihood of platelet aggregation in this proximity [65]. 


\subsection{Key findings}

- Steady-state simulations, driven by a constant inflow velocity equal to the peak systolic velocity (PSV) of the measured pulsatile flow, underestimated $\mathrm{SSR}_{\max }$ by $\sim 9 \%$ in pristine, and $\sim 19 \%$ in sutured vessels compared with a realistic pulse.

- The presence of sutures increases $\mathrm{SSR}_{\max }$ at suture sites by approximately 2.3fold in realistic pulsatile flow simulations, and 2.1-fold in steady-state simulations, when compared to a pristine vessel. The sutures also increase background SSR of the surrounding anastomotic region by $\sim 60 \%$.

- Shear gradients of $\sim 7.9 \times 10^{6} \mathrm{~m}^{-1} \mathrm{~s}^{-1}$ are seen at suture sites in pulsatile simulations. This may indicate increased strength of platelet membrane tethering and formation of aggregates, leading to thrombosis.

\subsection{Future directions}

This study also highlights areas for future work, in particular, simulation of blood as a non-Newtonian fluid and the compliant nature of blood vessel walls, as neither of these were considered here. Aspects such as fluid-structure interactions, anomalies in vessel geometry such as vessel wall bunching, which occurs when large suture bites are pulled tight causing an uneven wrinkled lumenal surface, vessel branches, or curves could also be incorporated. It is the aim of our group to incorporate these factors into microanastomotic simulations such that a clinically applicable model can be developed.

\section{Conclusions}

Steady-state simulations at PSV represent an approximation for microarterial blood flow modelling but significantly underestimates SSR, particularly in sutured vessels. Sinusoidal flows are a small (1-2\%) improvement on the steady-state approximations, but still values of $\mathrm{SSR}_{\max }$ are underestimated. We would recommend using a measured arterial waveform for simulating flows of this nature.

Further work to include the consideration of vascular wall compliance and fluidstructural interactions will be key to understanding the local haemodynamics in the most clinically relevant simulations.

\section{Acknowledgements}

The authors wish to express their thanks to the members of the Multi-scale Biology Study Group, University of Birmingham (12-15th December 2016) for their valuable discussions regarding this subject. The authors would also like to thank the Vascular Studies Unit at the University Hospital of South Manchester NHS Foundation Trust, for providing anonymised vascular data for creation of inlet profiles and geometries.

\section{Conflict of interest}

All authors declare they have no conflicts of interest. 


\section{Funding}

R A J Wain is a Research Fellow funded by the Institute of Translational Medicine, University of Birmingham, B15 2TT, UK.

D J Smith is funded by EPSRC Healthcare Technologies Challenge Award (EP/N021096/1).

The Multi-scale Biology Study Group, University of Birmingham (12-15th December 2016) was jointly funded by POEMS (Predictive modelling for healthcare technology through maths - EP/L001101/1) and MSB-Net (UK Multi-Scale Biology Network $\mathrm{BB} / \mathrm{M} 025888 / 1)$.

\section{Contribution}

All authors contributed to the design of the research, analysis of the data, and writing of this manuscript. RW constructed the geometries, performed the simulations, analysed the data, and compiled the manuscript. DS and JW provided technical assistance, mathematical support and, along with $\mathrm{DH}$, overall supervision of the project.

\section{Supplementary online content}

A series of animations are available to demonstrate the influence of transient inlet conditions on sutured microarterial anastomoses. In addition, data pertaining to the measured arterial waveform is freely available for use by other researchers via the University of Birmingham ePapers Repository:

http://epapers.bham.ac.uk/2981

\section{References}

[1] S. S. Kroll, M. A. Schusterman, G. P. Reece, M. J. Miller, G. R. Evans, G. L. Robb, B. J. Baldwin, Choice of flap and incidence of free flap success, Plastic and Reconstructive Surgery 98 (3) (1996) 459-463.

[2] R. K. Khouri, B. C. Cooley, A. R. Kunselman, J. R. Landis, P. Yeramian, D. Ingram, N. Natarajan, C. O. Benes, C. Wallemark, A prospective study of microvascular free-flap surgery and outcome, Plastic and Reconstructive Surgery 102 (3) (1998) $711-721$.

[3] J. Beugels, L. T. Hoekstra, S. M. H. Tuinder, E. M. Heuts, R. R. W. J. van der Hulst, A. A. Piatkowski, Complications in unilateral versus bilateral deep inferior epigastric artery perforator flap breast reconstructions: A multicentre study, Journal of Plastic, Reconstructive \& Aesthetic Surgery 69 (9) (2016) 1291-1298.

[4] C. L. Bendon, H. P. Giele, Success of free flap anastomoses performed within the zone of trauma in acute lower limb reconstruction, Journal of Plastic, Reconstructive \& Aesthetic Surgery 69 (7) (2016) 888-893.

[5] L. Bellidenty, R. Chastel, I. Pluvy, J. Pauchot, Y. Tropet, Emergency free flap in reconstruction of the lower limb. thirty-five years of experience, Annales De Chirurgie Plastique Et Esthetique 59 (1) (2014) 35-41. 
[6] H. R. Baumgartner, K. S. Sakariassen, Factors controlling thrombus formation on arterial lesions, Annals of the New York Academy of Sciences 454 (1) (1985) 162177.

[7] K. S. Sakariassen, L. Orning, V. T. Turitto, The impact of blood shear rate on arterial thrombus formation, Future Science 1 (4) (2015).

[8] S. Jandali, L. C. Wu, S. J. Vega, S. J. Kovach, J. M. Serletti, 1000 consecutive venous anastomoses using the microvascular anastomotic coupler in breast reconstruction, Plastic and Reconstructive Surgery 125 (3) (2010) 792-798.

[9] W. M. Rozen, I. S. Whitaker, R. Acosta, Venous Coupler for Free-Flap Anastomosis: Outcomes of 1,000 Cases, Anticancer Research 30 (4) (2010) 1293-1294.

[10] N. Chernichenko, D. A. Ross, J. Shin, J. Y. Chow, C. T. Sasaki, S. Ariyan, Arterial coupling for microvascular free tissue transfer, Otolaryngol. Head Neck Surg. 138 (5) (2008) 614-618.

[11] J. A. Spector, L. B. Draper, J. P. Levine, C. Y. Ahn, Routine use of microvascular coupling device for arterial anastomosis in breast reconstruction, Annals of Plastic Surgery 56 (4) (2006) 365-368.

[12] C. Y. Ahn, W. W. Shaw, S. Berns, B. L. Markowitz, Clinical experience with the $3 \mathrm{~m}$ microvascular coupling anastomotic device in 100 free-tissue transfers, Plastic and Reconstructive Surgery 93 (7) (1994) 1481-1484.

[13] M. S. Alghoul, C. R. Gordon, R. Yetman, G. M. Buncke, M. Siemionow, A. M. Afifi, W. K. Moon, From simple interrupted to complex spiral: a systematic review of various suture techniques for microvascular anastomoses, Microsurgery 31 (1) (2011) $72-80$.

[14] J. F. Mustard, E. A. Murphy, H. C. Rowsell, H. G. Downie, Factors influencing thrombus formation in vivo, The American Journal of Medicine 33 (5) (1962) 621647.

[15] G. D. O. Lowe, Virchow's triad revisited: abnormal flow, Pathophysiology of Haemostasis and Thrombosis 33 (5-6) (2003) 455-457.

[16] G. J. Roth, Developing relationships: arterial platelet adhesion, glycoprotein Ib, and leucine-rich glycoproteins, Blood 77 (1) (1991) 5-19.

[17] M. H. Kroll, J. D. Hellums, L. V. McIntire, A. I. Schafer, J. L. Moake, Platelets and shear stress, Blood 88 (5) (1996) 1525-1541.

[18] J. J. Hathcock, Flow effects on coagulation and thrombosis, Arteriosclerosis, Thrombosis, and Vascular Biology 26 (8) (2006) 1729-1737.

[19] F. Shen, C. J. Kastrup, Y. Liu, R. F. Ismagilov, Threshold response of initiation of blood coagulation by tissue factor in patterned microfluidic capillaries is controlled by shear rate, Arteriosclerosis, Thrombosis, and Vascular Biology 28 (11) (2008) 2035-2041. 
[20] K. S. Sakariassen, P. F. Nievelstein, B. S. Coller, J. J. Sixma, The role of platelet membrane glycoproteins Ib and IIb-IIIa in platelet adherence to human artery subendothelium, British Journal of Haematology 63 (4) (1986) 681-691.

[21] E. F. Grabowski, Platelet aggregation in flowing blood at a site of injury to an endothelial cell monolayer: quantitation and real-time imaging with the TAB monoclonal antibody, Blood 75 (2) (1990) 390-398.

[22] H. J. Weiss, V. T. Turitto, H. R. Baumgartner, Role of shear rate and platelets in promoting fibrin formation on rabbit subendothelium. Studies utilizing patients with quantitative and qualitative platelet defects, The Journal of Clinical Investigation 78 (4) (1986) 1072-1082.

[23] J. Jesty, W. Yin, P. Perrotta, D. Bluestein, Platelet activation in a circulating flow loop: combined effects of shear stress and exposure time, Platelets 14 (3) (2003) $143-149$.

[24] W. S. Nesbitt, E. Westein, F. J. Tovar-Lopez, E. Tolouei, A. Mitchell, J. Fu, J. Carberry, A. Fouras, S. P. Jackson, A shear gradient-dependent platelet aggregation mechanism drives thrombus formation, Nature Medicine 15 (6) (2009) 665-673.

[25] M. J. Maxwell, E. Westein, W. S. Nesbitt, S. Giuliano, S. M. Dopheide, S. P. Jackson, Identification of a 2-stage platelet aggregation process mediating sheardependent thrombus formation, Blood 109 (2) (2007) 566-576.

[26] R. A. J. Wain, J. P. M. Whitty, M. D. Dalal, M. C. Holmes, W. Ahmed, Blood flow through sutured and coupled microvascular anastomoses: a comparative computational study, Journal of Plastic, Reconstructive \& Aesthetic Surgery 67 (7) (2014) 951-959.

[27] R. A. J. Wain, D. Hammond, M. McPhillips, J. P. M. Whitty, W. Ahmed, Microarterial anastomoses: A parameterised computational study examining the effect of suture position on intravascular blood flow, Microvascular Research 105 (2016) 141148.

[28] J. P. M. Whitty, R. A. J. Wain, A. Fsadni, J. Francis, Computational NonNewtonian Hemodynamics of Small Vessels, Journal of Bioinformatics Computational and Systems Biology 1 (1) (2016) 103.

[29] G. S. Karanasiou, D. A. Gatsios, M. G. Lykissas, K. A. Stefanou, G. A. Rigas, I. E. Lagaris, I. P. Kostas-Agnantis, I. Gkiatas, A. E. Beris, D. I. Fotiadis, Modeling of blood flow through sutured micro-vascular anastomoses, in: Engineering in Medicine and Biology Society, 2015 37th Annual International Conference of the IEEE, 2015, pp. 1877-1880.

[30] K. Perktold, H. Florian, D. Hilbert, Analysis of pulsatile blood flow: a carotid siphon model, Journal of Biomedical Engineering 9 (1) (1987) 46-53.

[31] F. Migliavacca, G. Dubini, Computational modeling of vascular anastomoses, Biomechanics and Modeling in Mechanobiology 3 (4) (2005) 235-250. 
[32] C. A. Taylor, D. A. Steinman, Image-based modeling of blood flow and vessel wall dynamics: applications, methods and future directions: Sixth International BioFluid Mechanics Symposium and Workshop, March 28-30, 2008 Pasadena, California, Annals of Biomedical Engineering 38 (3) (2010) 1188-1203.

[33] D. A. Steinman, Assumptions in modelling of large artery hemodynamics, in: D. Ambrosi, A. Quarteroni, G. Rozza (Eds.), Modeling of Physiological Flows, Springer Milan, 2012, pp. 1-18.

[34] J. Al-Sukhun, C. Lindqvist, N. Ashammakhi, H. Penttilä, Microvascular stress analysis. Part I: simulation of microvascular anastomoses using finite element analysis, The British Journal of Oral \& Maxillofacial Surgery 45 (2) (2007) 130-137.

[35] R. F. Rickard, C. Meyer, D. A. Hudson, Computational modeling of microarterial anastomoses with size discrepancy (small-to-large), The Journal of Surgical Research 153 (1) (2009) 1-11.

[36] J. Al-Sukhun, H. Penttilä, N. Ashammakhi, Microvascular stress analysis: Part II. Effects of vascular wall compliance on blood flow at the graft/recipient vessel junction, The Journal of Craniofacial Surgery 22 (3) (2011) 883-887.

[37] E. Tenekecioglu, R. Torii, C. V. Bourantas, R. Cavalcante, Y. Sotomi, Y. Zeng, C. Collet, T. Crake, A. Abizaid, Y. Onuma, S. Su, T. Santoso, P. W. Serruys, Hemodynamic analysis of a novel bioresorbable scaffold in porcine coronary artery model, Catheterization and Cardiovascular Interventions 00 (2017) 1-8.

[38] M. Aletti, J.-F. Gerbeau, D. Lombardi, Modeling autoregulation in threedimensional simulations of retinal hemodynamics, Journal for Modeling in Ophthalmology 1.

[39] P. Perdikaris, L. Grinberg, G. E. Karniadakis, Multiscale modeling and simulation of brain blood flow, Physics of Fluids 28 (2) (2016) 021304.

[40] A. Kanaris, A. Anastasiou, S. Paras, Modeling the effect of blood viscosity on hemodynamic factors in a small bifurcated artery, Chemical Engineering Science 71 (2012) 202-211.

[41] C. L. Feldman, O. J. Ilegbusi, Z. Hu, R. Nesto, S. Waxman, P. H. Stone, Determination of in vivo velocity and endothelial shear stress patterns with phasic flow in human coronary arteries: A methodology to predict progression of coronary atherosclerosis, American Heart Journal 143 (6) (2002) 931-939.

[42] I. V. Pivkin, P. D. Richardson, G. Karniadakis, Blood flow velocity effects and role of activation delay time on growth and form of platelet thrombi, Proceedings of the National Academy of Sciences 103 (46) (2006) 17164-17169.

[43] C. Karmonik, J. Bismuth, M. G. Davies, A. B. Lumsden, Computational fluid dynamics as a tool for visualizing hemodynamic flow patterns, Methodist DeBakey Cardiovascular Journal 5 (3) (2009) 26-33. 
[44] G. Berti, Aneurist D23v2 - Analysis Protocols Version 2, Tech. Rep. v1.2, Integrated biomedical informatics for the management of cerebral aneurysms (2010).

URL http://www . aneurist.org

[45] A. Chaniotis, L. Kaiktsis, D. Katritsis, E. Efstathopoulos, I. Pantos, V. Marmarellis, Computational study of pulsatile blood flow in prototype vessel geometries of coronary segments, Physica Medica 26 (3) (2010) 140-156.

[46] ANSYS, Introduction to ANSYS CFX 16.0 - Lecture 4: Domains, Boundary Conditions and Sources, Tech. rep., ANSYS, Inc. (2015).

URL https://support.ansys.com

[47] F. M. White, Fluid Mechanics, 6th Edition, Pages 3-48, McGraw-Hill Higher Education, New York, NY, 2009.

[48] T. Sexl, Über den von EG Richardson entdeckten „Annulareffekt “, Zeitschrift für Physik 61 (5) (1930) 349-362.

[49] F. White, Viscous Fluid Flow, 3rd Edition, Pages 104-211, McGraw-Hill Education, New York, NY, 2005.

[50] D. N. Ku, D. P. Giddens, C. K. Zarins, S. Glagov, Pulsatile flow and atherosclerosis in the human carotid bifurcation. Positive correlation between plaque location and low oscillating shear stress, Arteriosclerosis 5 (3) (1985) 293-302.

[51] K. Perktold, M. Resch, R. O. Peter, Three-dimensional numerical analysis of pulsatile flow and wall shear stress in the carotid artery bifurcation, Journal of Biomechanics 24 (6) (1991) 409-420.

[52] S. Avril, J. M. Hunthley, R. Cusack, In vivo measurements of blood viscosity and wall stiffness in the carotid using PC-MRI, Revue Européenne de Mécanique Numérique 18 (1) (2009) 9-20.

[53] M. H. Friedman, G. M. Hutchins, C. B. Bargeron, O. J. Deters, F. F. Mark, Correlation between intimal thickness and fluid shear in human arteries, Atherosclerosis 39 (3) (1981) 425-436.

[54] K. S. Sakariassen, R. Joss, R. Muggli, H. Kuhn, T. B. Tschopp, H. Sage, H. R. Baumgartner, Collagen type III induced ex vivo thrombogenesis in humans. Role of platelets and leukocytes in deposition of fibrin., Arteriosclerosis, Thrombosis, and Vascular Biology 10 (2) (1990) 276-284.

[55] J. P. Bossavy, K. S. Sakariassen, A. Barret, B. Boneu, Y. Cadroy, A new method for quantifying platelet deposition in flowing native blood in an ex vivo model of human thrombogenesis, Thrombosis and Haemostasis 79 (1) (1998) 162-168.

[56] R. M. Barstad, H. E. Roald, Y. Cui, V. T. Turitto, K. S. Sakariassen, A perfusion chamber developed to investigate thrombus formation and shear profiles in flowing native human blood at the apex of well-defined stenoses., Arteriosclerosis, Thrombosis, and Vascular Biology 14 (12) (1994) 1984-1991. 
[57] R. M. Barstad, P. Kierulf, K. S. Sakariassen, Collagen induced thrombus formation at the apex of eccentric stenoses-a time course study with non-anticoagulated human blood, Thrombosis and Haemostasis 75 (4) (1996) 685-692.

[58] D. L. Bark, D. N. Ku, Platelet Transport Rates and Binding Kinetics at High Shear over a Thrombus, Biophysical Journal 105 (2) (2013) 502-511.

[59] K. S. Sakariassen, P. A. Aarts, P. G. de Groot, W. P. Houdijk, J. J. Sixma, A perfusion chamber developed to investigate platelet interaction in flowing blood with human vessel wall cells, their extracellular matrix, and purified components, The Journal of Laboratory and Clinical Medicine 102 (4) (1983) 522-535.

[60] J. M. Jiménez, P. F. Davies, Hemodynamically Driven Stent Strut Design, Annals of biomedical engineering 37 (8) (2009) 1483.

[61] J. Murphy, F. Boyle, Predicting neointimal hyperplasia in stented arteries using time-dependant computational fluid dynamics: A review, Computers in Biology and Medicine 40 (4) (2010) 408-418.

[62] D. M. Martin, E. A. Murphy, F. J. Boyle, Computational fluid dynamics analysis of balloon-expandable coronary stents: Influence of stent and vessel deformation, Medical Engineering \& Physics 36 (8) (2014) 1047-1056.

[63] F. J. Gijsen, J. C. Schuurbiers, A. G. van de Giessen, M. Schaap, A. F. van der Steen, J. J. Wentzel, 3d reconstruction techniques of human coronary bifurcations for shear stress computations, Journal of Biomechanics 47 (1) (2014) 39-43.

[64] E. Tenekecioglu, R. Torii, C. Bourantas, T. Crake, Z. Yaping, Y. Sotomi, Y. Onuma, M. Yılmaz, T. Santoso, P. Serruys, Preclinical assessment of the endothelial shear stress in porcine-based models following implantation of two different bioresorbable scaffolds: effect of scaffold design on the local haemodynamic micro-environment, EuroIntervention 12 (10) (2016) 1296.

[65] S. C. Shadden, S. Hendabadi, Potential fluid mechanic pathways of platelet activation, Biomechanics and Modeling in Mechanobiology 12 (3) (2013) 467-474. 